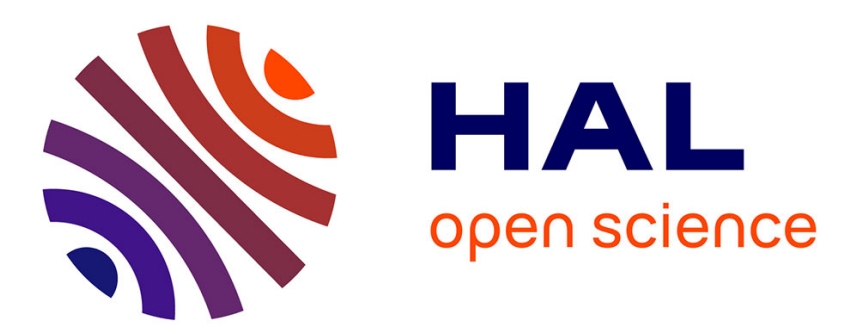

\title{
Caspase-3 triggers early synaptic dysfunction in a mouse model of Alzheimer's Disease
}

\author{
Marcello d'Amelio, Virve Cavallucci, Silvia Middei, Cristina Marchetti, \\ Simone Pacioni, Alberto Ferri, Adamo Diamantini, Daniela de Zio, Paolo \\ Carrara, Luca Battistini, et al.
}

\section{To cite this version:}

Marcello d'Amelio, Virve Cavallucci, Silvia Middei, Cristina Marchetti, Simone Pacioni, et al.. Caspase-3 triggers early synaptic dysfunction in a mouse model of Alzheimer's Disease. Nature Neuroscience, 2010, 10.1038/nn.2709 . hal-00599967

\section{HAL Id: hal-00599967 https://hal.science/hal-00599967}

Submitted on 12 Jun 2011

HAL is a multi-disciplinary open access archive for the deposit and dissemination of scientific research documents, whether they are published or not. The documents may come from teaching and research institutions in France or abroad, or from public or private research centers.
L'archive ouverte pluridisciplinaire HAL, est destinée au dépôt et à la diffusion de documents scientifiques de niveau recherche, publiés ou non, émanant des établissements d'enseignement et de recherche français ou étrangers, des laboratoires publics ou privés. 


\title{
Caspase-3 triggers early synaptic dysfunction in a mouse model of
}

\author{
Alzheimer's Disease \\ Marcello D’Amelio ${ }^{1,2}$, Virve Cavallucci ${ }^{1}$, Silvia Middei $^{3}$, Cristina Marchetti ${ }^{4}$, Simone Pacioni ${ }^{5}$, \\ Alberto Ferri ${ }^{6}$, Adamo Diamantini ${ }^{7}$, Daniela De Zio ${ }^{9}$, Paolo Carrara ${ }^{8}$, \\ Luca Battistini $^{7}$, Sandra Moreno ${ }^{8}$, Alberto Bacci $^{5}$, Martine Ammassari-Teule ${ }^{3}$, Hélène Marie ${ }^{4}$ \\ and Francesco Cecconi ${ }^{1,9}$
}

${ }^{1}$ Dulbecco Telethon Institute Laboratory of Molecular Neuroembryology IRCCS Fondazione Santa Lucia, 00143 Rome, Italy; ${ }^{2}$ Laboratory of Molecular Neuroscience, University Campus-Biomedico, 00128 Rome, Italy; ${ }^{3}$ CNR Institute of Neuroscience IRCCS Fondazione Santa Lucia, 00143 Rome, Italy; ${ }^{4}$ Laboratory of Molecular Mechanisms of Synaptic Plasticity, and, ${ }^{5}$ Laboratory of Cellular Physiology of Cortical Microcircuits, European Brain Research Institute, 00143 Rome, Italy; ${ }^{6} \mathrm{CNR}$ Institute of Neuroscience, Department of Psychobiology and Psychopharmacology, 00143 Rome, Italy; ${ }^{7}$ Laboratory of Neuroimmunology, IRCCS Fondazione Santa Lucia, 00143 Rome, Italy; ${ }^{8}$ Department of Biology-LIME, University Roma Tre, 00146 Rome, Italy; ${ }^{9}$ Department of Biology, University of Rome 'Tor Vergata', 00133 Rome, Italy.

Correspondence to: F.C.: francesco.cecconi@uniroma2.it

Running title: Caspase-3 in Alzheimer's Disease

Keywords: AMPA receptor, GluR1, Tg2576 mouse model, APPswe, calcineurin, cell death, synaptic degeneration, synaptic plasticity, memory function. 


\section{Summary}

Synaptic loss is the best pathological correlate of the cognitive decline in Alzheimer's Disease; yet, the molecular mechanisms underlying synaptic failure are unknown. Here we report a non-apoptotic baseline caspase-3 activity in hippocampal dendritic spines, and an enhancement of this activity at the onset of memory decline in the Tg2576-APPswe mouse model of Alzheimer's Disease. We show that, in spines, caspase-3 activates calcineurin which, in turn, triggers dephosphorylation and removal of the GluR1 subunit of AMPA-type receptor from post-synaptic sites. These molecular modifications lead to alterations of glutamatergic synaptic transmission and plasticity, and correlate with spine degeneration and a deficit in hippocampal-dependent memory. Importantly, pharmacological inhibition of caspase-3 activity in $\operatorname{Tg} 2576$ mice rescues the observed Alzheimerlike phenotypes. Therefore, we identify a novel caspase-3-dependent mechanism driving synaptic failure and contributing to cognitive dysfunction in Alzheimer's Disease. These findings point to caspase-3 as possible avenues for pharmacological therapy during early disease stages.

Episodic hippocampal-dependent memory loss, the earliest clinical sign of Alzheimer's disease, is thought to be due to changes in synaptic function rather than neuronal loss ${ }^{1,2}$. Specifically, functional brain imaging studies revealed hippocampal mild abnormalities prior to clinical diagnosis and in the absence of structural brain atrophy, suggesting an altered functional connectivity of hippocampus at early stages of disease $\mathrm{s}^{3-5}$.

Dendritic spines are likely to be the first affected synaptic elements during early cognitive decline ${ }^{6}$. This is supported by several lines of evidence, such as: i) hippocampal spine-mediated plasticity underlies learning and memory ${ }^{7}$; ii) post-mortem hippocampus from Alzheimer patients shows a significant decrease in dendritic spine density compared to age-matched controls ${ }^{8}$ and iii) transgenic 
mice expressing mutated forms of the amyloid precursor protein (APP), associated with familial Alzheimer's Disease, show age-dependent reductions in spine density, prior to plaque deposition ${ }^{9}$. Nevertheless, the molecular link between APP mutations triggering Alzheimer's Disease, and the occurrence of early spine loss remains elusive. Interestingly, a localized caspase-3 activity, causing synaptic failure, has been observed in vitro ${ }^{10}$, but the molecular mechanism linking caspase-3 activity to synaptic loss is far from being elucidated.

Here, we analyzed caspase- 3 activity in hippocampal synapses of the $\operatorname{Tg} 2576$ transgenic mouse model, harboring the human APPswe mutant allele linked to familial Alzheimer's Disease ${ }^{11}$. These mice develop early synaptic deficits ${ }^{12}$ and several neuropathological features at older age, including amyloid plaques and dystrophic neurites ${ }^{13}$. Although Tg2576 mice lack neurofibrillary tangles and significant neuronal $\operatorname{loss}^{14}$, there is strong evidence that accumulation of the amyloid- $\beta$ (A $\beta$ ) peptide, derived via APP proteolysis, is responsible for age-related memory decline in this model ${ }^{15-}$ 17.

By means of several experimental approaches, we demonstrate an increase of caspase- 3 activity in Tg2576 hippocampal synapses at 3 months of age, much earlier than amyloid plaque deposition is detectable $^{11}$. We report that this enhanced local caspase-3 activity leads to a permanent activation of calcineurin which causes, in turn, AMPA receptor (AMPAR) GluR1 subunit dephosphorylation and its removal from the post-synaptic sites. Furthermore, we show that this caspase- 3 activity increase is matched by i) a deficit in hippocampal-dependent contextual fear conditioning (CFC), ii) a reduction of spine density in CA1 pyramidal neurons, iii) an alteration in basic glutamatergic synaptic transmission and enhanced LTD in these same neurons. Importantly, we demonstrate that pharmacological inhibition of caspase-3 activity in $\operatorname{Tg} 2576$ mice restores post-synaptic densities (PSD) composition, glutamatergic synaptic transmission and spine size, and provides a partial rescue of memory during CFC. Collectively, our data indicate that caspase-3 activity plays a crucial role in the early synaptic dysfunction reported during Alzheimer pathology in $\operatorname{Tg} 2576$ mice. 


\section{Results}

\section{Onset of memory decline in Tg2576 mice}

We evaluated hippocampal-dependent memory in Tg2576 mice and their wild-type controls by means of the CFC task ${ }^{18,19}$. We observed that, although wild-type and Tg2576 mice exhibit similar baseline activity during the training trials before the shock delivery (Supplementary Fig. 1), memory performance, as assessed by freezing behavior upon re-exposure of mice to the context where they were previously shocked, was reduced in $\mathrm{Tg} 2576$ mice compared to wild-type mice (Fig. 1a). Notably, this effect reached statistical significance at 3 but not at 2 months of age, suggesting the 3-month age-point as the onset of hippocampal-dependent memory impairment.

To assess whether early cognitive decline in $\operatorname{Tg} 2576$ mice was associated with dendritic spine alterations, we measured spine density and spine size in Golgi-stained CA1 pyramidal neurons of the dorsal hippocampus in 2- and 3-month-old wild-type and Tg2576 mice (Fig. 1b). Number of spines along basal dendrites laying in the stratum oriens is unaltered in Tg2576 mice at both 2 and 3 months of age. We detected on apical dendrites laying in the stratum radiatum a significant reduction in spine density in 3-month-old $\mathrm{Tg} 2576$ mice compared to both wild-type littermate and 2-month-old Tg2576 mice (Fig 1c). Along the same apical dendrites, Tg2576 mice exhibited a higher proportion of spines with smaller head diameters (Fig. 1d) and the total dendrite length was also significantly reduced (Fig. 1e) in 3-month-old Tg2576 mice (1429.10 $\pm 97.10 \mu \mathrm{m})$ compared to age-matched wild-type mice $(2057.99 \pm 195.30 \mu \mathrm{m})$. Altogether, these alterations result in a reduced synaptic input in the hippocampus of $\operatorname{Tg} 2576$ mice that likely contributes to their cognitive impairment.

\section{Detection of early synaptic deficits in Tg2576 mice}

Structural modifications in the PSD precede morphological alterations of the dendritic spine ${ }^{20}$. In order to investigate the molecular basis of dendritic spine loss in $\mathrm{Tg} 2576$ mice we thus evaluated the PSD composition in wild-type and Tg2576 mice at 3 months of age. Isolated PSD preparation 
extracts from hippocampi of $\operatorname{Tg} 2576$ and age-matched wild-type mice were blotted and probed with antibodies against the main PSD components (Fig. 2a and Supplementary Fig. 2a,b). Whereas the relative amounts of most post-synaptic components remained essentially unchanged in PSD from both genotypes, the levels of the AMPAR subunit GluR1 and one of its phosphorylated form (GluR1pSer845) were significantly reduced in the PSD obtained from Tg2576 mice compared to wild-type (Fig. 2a). However, in total hippocampal homogenates, the GluR1 levels were unaffected while GluR1pSer845 levels were reduced in Tg2576 hippocampus (Fig. 2b). Furthermore, the subcellular distribution of GluR1 was also markedly changed in $\operatorname{Tg} 2576$ mice, with a significant decrease in the PSD-enriched fraction (TxP) and a significant increase in the microsome-enriched fraction (P3) (Fig. 2c and Supplementary Fig. 2c). Altogether, these results argue against a global degradation of GluR1 in the whole hippocampus of $\mathrm{Tg} 2576$ mice, but suggest a synaptic redistribution of GluR1 and specific dephosphorylation of its Ser845.

Since it has been shown that $A \beta$ impairs synaptic plasticity ${ }^{21}$, we asked whether modulating $A \beta$ load in Tg2576 mice could affect GluR1 distribution. We injected intra-hippocampally the $\gamma$ secretase inhibitor (DAPT) in 3-month-old Tg2576 mice and we found that there was a marked translocation of GluR1 to the PSD-enriched fraction in the DAPT-injected ipsilateral side, compared to the vehicle-injected controlateral hippocampus already $15 \mathrm{hrs}$ after injection (Supplementary Fig. 3a). We also found that the head diameter of dendritic spines was enlarged in the DAPT-injected ipsilateral side, compared to the vehicle-injected controlateral hippocampus (Supplementary Fig. 3b). Finally, bilateral injection of DAPT in the hippocampus of Tg2576 mice $15 \mathrm{hrs}$ before training significantly rescued memory deficits without affecting motor activity (Supplementary Fig. 3c,d). Together with previous in vitro studies ${ }^{22}$, these in vivo results indicate that $\mathrm{A} \beta$ contributes to synaptic modifications, since alterations in GluR1 distribution and dendritic spine alterations were blocked by $\gamma$-secretase-dependent APP processing inhibition.

Alterations of the PSD composition of hippocampal neurons in 3-month-old Tg2576 mice may lead to changes in glutamatergic synaptic transmission. We first evaluated basic synaptic transmission 
by measuring input-output relationships of field excitatory post-synaptic potentials (fEPSPs) of CA1 pyramidal neurons evoked by stimulation of the Schaffer collateral pathway from acute hippocampal slices obtained from wild-type and Tg2576 mice. No difference was detected between Tg2576 and wild-type mice using this analysis (Supplementary Fig. 4). To detect more subtle alterations in synaptic transmission we next performed whole-cell patch-clamp recordings of CA1 pyramidal neurons. As the main alteration in the PSD of 3-month-old Tg2576 mice was a downregulation of GluR1 levels whereas the levels of NMDA receptor (NMDAR) subunits remained relatively stable, we first assessed the relative contribution of AMPARs and NMDARs to evoked excitatory post-synaptic currents (EPSCs), measured as an AMPA/NMDA ratio. We found that this

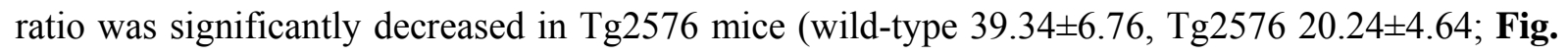
3a). We evaluated pre-synaptic release short-term plasticity by measuring the paired-pulse ratios (PPR) of synaptic responses. PPR did not differ in Tg2576 (1.42 \pm 0.19$)$ compared to wild-type (1.40 \pm 0.07$)$ mice, arguing against any alterations in pre-synaptic release properties at this age (Fig. 3b). We also examined AMPAR transmission by measuring spontaneous miniature excitatory postsynaptic currents (mEPSCs), which represent AMPAR-mediated post-synaptic responses to spontaneous release of individual glutamate vesicles (Fig. 3c,d). We did not observe a statistically significant difference in mEPSC amplitude in $\operatorname{Tg} 2576(12.77 \pm 0.66)$ with respect to wild-type (14.12 \pm 0.68$)$ mice, although a trend towards a decrease was apparent (Fig. 3d). We however observed that mEPSC frequency was significantly decreased in $\operatorname{Tg} 2576$ mice (wild-type $1.15 \pm 0.14$, Tg2576 0.80 \pm 0.09 ; Fig. 3d). A lack of PPR alterations together with a reduction of mEPSC frequency is consistent with a decrease in the number of AMPAR-containing synapses. These results are in line with the reduction of GluR1 levels in the PSD and a loss of spines observed in 3month-old Tg2576 mice, although we cannot exclude additional NMDAR alterations.

The role of GluR1 phosphorylation in synaptic plasticity paradigms, such as long term potentiation (LTP) and long term depression (LTD), has been extensively studied ${ }^{23}$. We therefore tested whether the specific reduction of GluR1pSer845 in the PSD affected LTP and LTD expression. Similar 
levels of synaptic potentiation were observed in wild-type and Tg2576 mice (Fig. 3e). This result suggests that the mechanisms of GluR1 trafficking to the PSD during LTP are still intact in Tg2576 mice at this age, as previously reported ${ }^{24}$.

By contrast, we found that the magnitude of LTD observed in Tg2576 mice was slightly, but significantly, enhanced compared to wild-type mice (Fig. 3f). These data suggest that the mechanisms driving synaptic depression, including specific dephosphorylation of GluR1Ser845, are more efficient in Tg2576 than in wild-type mice ${ }^{25}$.

\section{Active caspase-3 increases in Tg2576 hippocampal synapses}

By means of in vitro studies, a localized caspase-3-dependent apoptosis has been proposed to contribute to early synaptic loss in Alzheimer and other neurodegenerative diseases ${ }^{10}$; the mechanism(s) underlying this process, however, remains elusive.

We therefore tested whether caspase-3 activity was responsible for the observed PSD alteration and spine loss in the Tg2576 hippocampus. We compared caspase-3-like activity in whole cell extracts and synaptosomal fraction prepared from hippocampi of 2- and 3-month-old wild-type and $\mathrm{Tg} 2576$ mice. A significant increase in caspase-3 activity, in both total hippocampal homogenates and synaptosomes, was observed in $\operatorname{Tg} 2576$ mice at 3 but not at 2 months of age (Fig. 4a).

A significant increase in caspase-3 activity was also detected in Tg2576-derived synaptosomes when compared to those from age-matched wild-type mice at 6 and 18 months after birth (Supplementary Fig. 5).

The cleaved forms of caspase-3 were detected in synaptosomes showing a stronger immunoreactivity in Tg2576 compared to wild-type mice at 3 months of age (Fig. 4b). Intriguingly, we found that procaspase- 3 is also more abundant in the synaptosomal fraction, when compared to the total homogenate. These findings suggest that caspase-3 is preferentially localized and selectively activated within the synaptic compartment, whereas the other effector caspases (caspase7 and -6) remain uncleaved (Supplementary Fig. 6). 
To provide additional evidence of the synaptic localization of active caspase- 3 in the hippocampus, we performed pre-embedding immunoelectron microscopy, using an antibody specific to cleaved caspase-3. Immunostained spines were significantly more abundant in $\mathrm{Tg} 2576$ neurons than in their wild-type counterparts (Fig. 4c and Supplementary Fig. 7). Within the spine, the immunoprecipitates were specifically concentrated within the PSD. No immunoreactivity was found in cell soma, nuclei, pre-synaptic terminals, or surrounding glial projections.

The exposure of phosphatidylserine (PS) residue on the plasma membrane is usually considered as a downstream event of caspase-3 activation and a hallmark of apoptosis ${ }^{26}$. In order to examine apoptosis in hippocampal synapses, we analyzed the exposure of PS in the synaptosomal fraction where active caspase-3 accumulates. By labeling with annexin $\mathrm{V}$ and a viability marker (calcein AM) prior to flow cytometry, local synaptic death was measured in intact synaptosomes prepared from the hippocampus of 3-month-old wild-type and Tg2576 mice. We found that PS exposure is increased in Tg2576-derived synaptosomes when compared to wild-type (Fig. 4d). The absence of genotype-dependent difference in propidium iodide (PI) labeling of hippocampal cell nuclei, isolated from the same hippocampi used for synaptosome preparation, rules out the possibility of wide-spread neuronal apoptosis (Fig. 4e). The reported $\sim 5 \%$ of nuclei incorporating PI is merely due to the experimental procedure (background). Unstained controls are shown in Supplementary Fig. 8a,b. In line with these results, no cell death was detected by TUNEL assay in wild-type nor in Tg2576 hippocampi (Supplementary Fig. 8c).

An increase in caspase-3 activity in the absence of neuronal death suggests a sub-lethal mitochondrial stress. This is in line with the finding that, in the hippocampus of 3-month-old Tg2576 mice, protein oxidation level is significantly higher than in those from age-matched controls (Supplementary Fig. 9).

Comprehensively, these data demonstrate that oxidative stress is initiated in $\operatorname{Tg} 2576$ mice already at 3 months of age, synchronous with the occurrence of some apoptotic biochemical features which can occur, locally, at the synapses. 
The canonical activation of caspase-3 occurs in a wide range of cellular and in vivo systems by means of two main pathways ${ }^{27,28}$ : the extrinsic pathway of cell death, mediated by the death receptor family; and the mitochondrial (intrinsic) pathway of cell death, mediated by the Apaf1centered apoptosome. Since the main route of neuronal death is along the mitochondrion/apoptosome pathway, we expected caspase-3 to be activated via the intrinsic pathway. To confirm the involvement of the Apafl-centered apoptosome in this context, we used Tg2576-derived primary hippocampal cultured neurons lacking Apafl $\left(\mathrm{Tg}^{+} / \mathrm{Apafl}^{-/-}\right.$double mutants). In both total lysates and synaptosomes from $\mathrm{Tg}^{+} / \mathrm{Apaf1}^{-/-}$neurons, we did not observe caspase-3 cleavage (Supplementary Fig. 10a,b). In addition, in $\mathrm{Tg}^{+} / A p a f 1^{+/+}$and $\mathrm{Tg}^{+} / \mathrm{Apafl}^{-/-}$ neurons, caspase-7 and -6 were not activated (Supplementary Fig. 10c). These results suggest that, in $\operatorname{Tg} 2576$ neurons, caspase-3 activation is apoptosome-dependent.

\section{Caspase-3 mediates GluR1 removal from post-synaptic sites}

As caspase-3 activity was increased in the post-synaptic compartments and synaptic GluR1 trafficking was altered in Tg2576 hippocampal neurons, we tested whether these two molecules interacted epistatically. We performed direct fluorimetric measurement of caspase-3-like activity in acute hippocampal slices from 3-month-old Tg2576 mice after 2 hrs of incubation with the caspase3 inhibitor z-DEVD-fmk and we observed that z-DEVD-fmk inhibits caspase-3-like activity in a concentration-dependent manner (Fig. 5a). Notably, cleaved PARP (a nuclear substrate of caspase3) was undetectable in all experimental conditions, ruling out the possibility that z-DEVD-fmk protects neurons from cell death induced by slicing and incubation conditions (Supplementary

\section{Fig.11).}

Immunoblot analysis of PSD from Tg2576 slices treated with z-DEVD-fmk showed an increase of GluR1 and GluR1pSer845, compared to the vehicle-treated controls. By contrast, caspase-3 inhibition had no effect on NMDAR subunits and PSD-95 levels (Fig. 5b). A linear regression analysis showed that the GluR1 levels in the PSD are inversely correlated to caspase-3-like activity 
(Fig. 5c). Conversely, analysis of PSD from wild-type slices treated with z-DEVD-fmk showed that caspase-3 inhibition causes an inverse effect on GluR1 surface expression (Supplementary Fig. 12).

We also analyzed the content of GluR1 in total homogenate of $\mathrm{Tg} 2576$ hippocampal slices treated with z-DEVD-fmk compared to untreated slices. Caspase-3 inhibition caused an increase of GluR1pSer845 but no difference was observed in total GluR1 levels (Fig. 5d).

The treatment of $\operatorname{Tg} 2576$ hippocampal slices with the caspase negative control inhibiting other proteases, z-FA-fmk, caused an expected reduction of cathepsin B activity but it did not affect the phosphorylation of GluR1 (Supplementary Fig. 13a,b). Comprehensively, these results indicate that caspase-3, under these experimental conditions, is specifically involved in GluR1 phosphorylation and mobility, but not degradation.

To confirm the involvement of caspase- 3 in the regulation of synaptic transmission, we measured AMPA/NMDA ratio of CA1 neurons in acute Tg2576 hippocampal slices after 2 hrs of incubation with caspase-3 inhibitor or vehicle. We found that the AMPA/NMDA ratio in z-DEVD-fmk-treated slices was significantly increased with respect to vehicle-incubated slices restoring the ratio to wildtype value (vehicle control 19.46 \pm 2.77 , z-DEVD-fmk 46.43 \pm 8.48 , wild-type $39.34 \pm 6.76$; Fig. 5e). PPR of synaptic responses did not differ in z-DEVD-fmk-treated compared to vehicle-treated slices (vehicle control 1.34 \pm 0.05 , z-DEVD-fmk $1.32 \pm 0.05$, wild-type $1.40 \pm 0.07$; Fig. 5f), indicating that caspase-3 inhibition does not affect pre-synaptic release properties. These data suggest that blocking caspase-3 activity in $\operatorname{Tg} 2576$ neurons is sufficient to specifically restore post-synaptic glutamate transmission to wild-type levels.

As calcineurin is required for $A \beta$-mediated synaptic removal of AMPAR, leading to loss of dendritic spines ${ }^{29}$, and calcineurin A (CN-A) is known to be proteolytically activated by caspase- $3^{30}$, we hypothesized that hippocampal CN-A activity would increase upon caspase-3 cleavage thus inducing GluR1 removal from the PSD. To test whether CN-A is a hippocampal caspase-3 substrate, we performed an immunoblot analysis of the total homogenate from $\mathrm{Tg} 2576$ slices treated with z- 
DEVD-fmk or the vehicle control by using an antibody specific to the N-terminal of CN-A. We found that CN-A processing is reduced in Tg2576 slices treated with z-DEVD-fmk (Fig. 6a). Given that the $45 \mathrm{kDa} \mathrm{CN}-\mathrm{A}$ fragment produced by caspase-3 cleavage contains the whole catalytic domain but lacks the autoinhibitory one, we assayed $\mathrm{CN}$-A activity in response to caspase-3 inhibition. We measured CN-A activity in a calcium-free medium, to exclude a calcium-dependent regulation of $\mathrm{CN}-\mathrm{A}$ activation. In addition, the specificity of the activity assay was confirmed by using the calcineurin inhibitor FK506 (Fig. 6b). We observed a significant reduction of calciumindependent $\mathrm{CN}$-A activity in z-DEVD-fmk-treated slices, as compared to vehicle-treated slices, indicating that caspase-3-dependent cleavage of $\mathrm{CN}-\mathrm{A}$ causes its activation.

To confirm the effect of calcineurin activity on GluR1 phosporylation we also analyzed $\operatorname{Tg} 2576$ hippocampal slices treated with FK506 compared to untreated slices. Incubation with calcineurin inhibitor for 2 hrs caused an increase of GluR1pSer845 but no differences were observed in total GluR1 from Tg2576 slices compared to vehicle-treated controls (Supplementary Fig.14).

In addition, caspase-3 activity increase in 3-month-old Tg2576 hippocampus was associated in vivo with an increase of both activity and processing of calcineurin when compared to wild-type (Supplementary Fig. 15a,b). The levels of active calpain-1 were similar in young wild-type and Tg2576 mice, even though an increase of calpain-1 cleavage was observed in older mice (Supplementary Fig. 15c). This result excludes the involvement of calpain-1 in CN-A processing at this very early stage of disease in $\operatorname{Tg} 2576$ mouse model.

Taken together, these results highlight a role for caspase-3 in GluR1 synaptic removal via CN-A cleavage.

\section{Caspase-3 inhibition in vivo rescues Tg2576 phenotype}

We next addressed the possibility of rescuing GluR1 PSD levels, spine size and memory deficits by in vivo caspase-3 inhibition. As z-DEVD-fmk cannot cross the blood brain barrier, and to minimize the pharmacological effect on other brain regions, we injected z-DEVD-fmk directly in one 
hippocampus and the vehicle in the controlateral hippocampus as control. Fifteen hours after injection, we observed a reduction of both caspase- 3 and calcineurin activity together with a reduction of calcineurin processing in the ipsilateral z-DEVD-fmk-injected side, compared to the controlateral vehicle-injected hippocampus (Fig. 7a,b). Then, we analyzed the synaptic GluR1 content, and we observed a marked translocation of GluR1 from the microsomal fraction to the PSD (Fig. 7c) together with an increase of GluR1pSer845 in the PSD (Supplementary Fig. 16). To confirm the effect of caspase-3 inhibition on GluR1 synaptic distribution we used the polyglutamic acid (PGA)-peptoid QM56 to inhibit the activity of the apoptosome ${ }^{31}$, a macromolecular complex activating caspase- 3 and the mitochondrial-dependent apoptosis pathway ${ }^{32}$.

Forty-eight hours after QM56 intra-hippocampal injection, we analyzed caspase-3 activity and GluR1 PSD levels. We observed a reduction of caspase-3 activity in the ipsilateral QM56-injected side, compared to the controlateral vehicle-injected hippocampus (Supplementary Fig. 17a) and, as reported by using z-DEVD-fmk, we observed a marked translocation of GluR1 from the microsomal fraction to the PSD, with an increase of GluR1pSer845 in the PSD (Supplementary Fig. 17b,c).

Since we previously showed that the DAPT injection in the hippocampus affected GluR1 synaptic distribution (Supplementary Fig. 3), we also assessed whether this effect was mediated by caspase- 3 by analyzing caspase-3 activity in Tg2576 hippocampus 15 hrs after DAPT injection. We observed a reduction of caspase-3 activity in the ipsilateral DAPT-injected side, compared to the controlateral vehicle-injected hippocampus (Supplementary Fig. 18).

Taken together, these results clearly prove i) the specificity of caspase- 3 inhibition on synaptic function; ii) the main route of caspase-3 activation is mitochondria-dependent.

We also found that the head diameter of dendritic spines was enlarged in the ipsilateral z-DEVDfmk-injected side compared to the controlateral vehicle-injected hippocampus restoring spine size to wild-type levels (Figs. 7d and 1c). Finally, bilateral injection of z-DEVD-fmk in the hippocampus of Tg2576 mice 15 hrs before training in CFC significantly rescued the memory deficits (Fig. 7e) 
without affecting motor activity (Supplementary Fig. 19). Interestingly, intra-hippocampal injection of z-DEVD-fmk caused an inverse effect on wild-type mice, confirming a physiological role of caspase-3 in hippocampal function. Specifically, synaptic fractionation of z-DEVD-fmktreated wild-type hippocampi demonstrated a reduction of GluR1 at PSD compared to vehicle controls (Supplementary Fig. 20). Moreover, z-DEVD-fmk-treated wild-type mice worsened memory in the CFC task (Fig. 7e).

\section{Discussion}

Caspase-3 activation is considered the terminal step in the biochemical cascade leading to apoptotic cell death ${ }^{33}$. However, the opinion that caspase-3 is more than just a 'killer' involved in neuronal programmed cell death is supported by studies implicating caspase-3 in regulation of synaptic plasticity $^{34-36}$.

Evidence for a role of caspase-3 in Alzheimer's Disease includes caspase-3 activation in A $\beta$-treated neurons ${ }^{37}$, immunodetection of activated caspase- 3 in patient brain ${ }^{38,39}$, involvement of caspase- 3 in APP proteolysis and A $\beta$ peptide formation ${ }^{38}$, increase of caspase- 3 in the PSD fractions of Alzheimer patients ${ }^{40}$.

Despite this exciting array of data indicating caspase-3 as an etiologic factor in Alzheimer's Disease, the molecular mechanism(s) linking Alzheimer-related synaptic dysfunction to caspase-3 activity remains elusive.

We now identify local up-regulation of caspase-3 in hippocampal CA1 dendritic spines as a key process triggering functional and structural hippocampal synaptic alterations correlating with the onset of memory deficits in the APPswe Tg2576 mouse model. Our combined ex-vivo and in vivo analyses provide strong evidence that Tg2576 mice display enhanced caspase-3 activity in dendritic spines, resulting in a down-regulation of the surface expression of GluR1-containing AMPAR via proteolytic activation of calcineurin. The alterations of PSD composition are accompanied by synaptic transmission deficits, enhanced LTD and reduced spine size and density. These data are 
supported by a study reporting that synaptic AMPAR removal is both necessary and sufficient for A $\beta$-induced spine $\operatorname{loss}^{31}$. The implication of this novel non-apoptotic caspase- 3 dependent mechanism at the onset of Alzheimer neurodegeneration is proved by ex-vivo and in vivo rescue experiments using the caspase-3 inhibitor z-DEVD-fmk. Indeed, we demonstrate that caspase-3 inhibition restores glutamatergic synaptic transmission in $\operatorname{Tg} 2576$ slices to wild-type levels and that, in vivo, a single injection of caspase-3 inhibitor is sufficient to down-regulate caspase-3 and calcineurin activity thus restoring GluR1 levels at PSD, spine size and memory performance. The pharmacological approach used in this work affords the unprecedented possibility of acutely reducing caspase- 3 activity at the onset of Alzheimer-related cognitive decline in the $\mathrm{Tg} 2576$ mouse model. Indeed, this approach avoids any interference with developmental parameters, postnatal differentiation and neuron physiology which could be perturbed by using genetic approaches, as highlighted by abnormal brain development in knock-out mice with an altered caspase-3 activation $^{41-44}$.

Our data strongly support the possibility of targeting caspase-3 activity to reverse hippocampal function deficits at the onset of Alzheimer pathology. Nevertheless, the opposite effects produced by in vivo injection of the caspase-3 inhibitor in wild-type mice suggest that therapeutic intervention should take into account the critical physiological role of caspase-3-dependent proteolysis in synaptic plasticity and memory, independent of its role in apoptosis.

Our proposed model explaining the role of caspase- 3 in dendritic spine degeneration is illustrated in Supplementary Fig. 21. Intraneuronal accumulation of $A \beta$ within distal processes and synaptic compartment $^{45}$ leads to the generation of free radicals ${ }^{46}$. These reactive species, in turn, disrupt the electron transport chain thus leading to mitochondrial dysfunction and consequent cytochrome $c$ release from the outer membrane. The release of cytochrome $c$ into the cytosol induces the formation of the apoptosome which, in turn, recruits and activates caspase-3. Proteolytic cleavage of calcineurin induces a loss of its calcium-calmodulin sensitivity ${ }^{32}$. This causes an increase in its phosphatase activity resulting in an increased dephosphorylation of GluR1 at Ser845, followed by 
AMPAR removal from PSD and progressive spine loss. However, the cleavage of other synaptic substrates by caspase- 3 cannot be excluded.

Consistent with our model, recent evidence identified the mitochondria as a key organelles in A $\beta$ induced dendritic spine loss and neuronal damage ${ }^{47}$. Here, we propose a mechanism downstream of mitochondrial dysfunction, which orchestrates dendritic spine shrinkage and loss during early Alzheimer-related neurodegeneration.

In conclusion, the above identified mechanism points to caspase- 3 as a possible early biomarker and should encourage further studies on its regulation to develop rationale "synaptoprotective" therapies for preventing or slowing down Alzheimer's Disease progression. 


\section{Acknowledgements}

We thank the Animal Facility of the IRCCS Fondazione Santa Lucia/EBRI/CNR for the mouse work, Taconic for the Tg2576 mice (NY), M. Acuña-Villa and M. W. Bennett for editorial and secretarial work, R. Nardacci, F. Fanelli, and M. Nencini for research assistance and help with image processing. A. Roberto and A. Pignataro for help with Neurolucida measurements. We are grateful to Dr. E. Pérez-Payá for providing us with apoptosome inhibitor QM56. This work was supported in part by grants from the Telethon Foundation, Ricerca Corrente and Ricerca Finalizzata from the Italian Ministry of Health, the Italian Ministry of University and Research, and Compagnia di San Paolo.

\section{Author contributions}

M.DA. and V.C. designed and carried out all molecular biology experiments and caspase-3 analysis. V.C. contributed to writing the manuscript. S.Mi. and M.T-A. performed behavioral and dendritic spine analysis. S.Mi. performed surgery. A.B. and S.P. performed LTP analysis. H.M. and C.M. performed patch-clamp and LTD experiments. A.F. carried out calcineurin activity assay. S.Mo. and P.C. performed immunoelectron microscopy analysis. L.B. and A.D. performed FACS analysis. D.D.Z. analyzed the oxidative stress.

M.DA. and F.C. conceived and designed the study, supervised all experiments and wrote the manuscript.

All authors discussed the results and commented on the manuscript. 


\section{Figure Legends}

Figure 1. Contextual Fear Conditioning (CFC) performance and morphology of CA1 neuron dendrites in Tg2576 and wild-type mice.

a, Percentage of freezing time during CFC test in wild-type (WT) and Tg2576 (Tg) mice at 2 and 3 months of age. Data are expressed as mean \pm s.e.m. ${ }^{* *} P=0.009$ ( $N=10$ mice for each age and genotype). b, Left panel: representative photomicrograph of a Golgi-stained section of the dorsal hippocampus. Scale bar, $100 \mu \mathrm{m}$. Right panels: representative segments of basal and apical dendrites of CA1 hippocampal neurons in 3-month-old wild-type and Tg2576 mice. Scale bar, 10 $\mu \mathrm{m}$. c, Spine density is expressed as the mean spine number per $1 \mu \mathrm{m}$ dendrite segment \pm s.e.m. $* P=0.010, * * * P=0.0007$ ( $N=6$ mice for each genotype at 2 months of age, $N=10$ wild-type mice at 3 months of age, $N=8 \mathrm{Tg} 2576$ mice at 3 months of age, $n=11$ neurons from each mouse). d, Cumulative frequencies of spine head diameters values in apical dendrites of pyramidal neurons. A shift of the curve to the left indicates that $\operatorname{Tg} 2576$ mice show a general reduction of spine head diameters. $P=0.039(N=5, n=1000$ spines from each mouse). e, Total dendritic length of CA1 hippocampal neurons. Data are presented as mean \pm s.e.m. $* * P=0.006(N=5, n=6$ neurons from each mouse).

\section{Figure 2. Three-month-old Tg2576 mice show altered hippocampal GluR1 distribution.}

a, Representative immunoblots of PSD proteins extracted from wild-type (WT) and Tg2576 (Tg) hippocampi probed with the indicated antibodies and densitometric quantification of changes in grey values expressed as mean ratio $(\mathrm{Tg} / \mathrm{WT}) \pm$ s.d. ${ }^{*} * P=0.006$ (GluR1), $* * P=0.003$ (GluR1pSer845) ( $N=5$ mice from each genotype). b, Representative immunoblots of hippocampal total protein extract probed with the indicated antibodies and densitometric quantification of changes in grey values expressed as mean \pm s.d. (wild-type is indicated as $100 \%)$. ${ }^{*} P=0.024(N=4)$. c, Representative GluR1 immunoblots of hippocampal synaptic fractionation. TxP, PSD-enriched fraction; P3, microsome-enriched fraction. Densitometric quantification of changes in grey values 
expressed as mean \pm s.d. (wild-type is indicated as $100 \%) . * P=0.041(N=4)$. For full-length blots see Supplementary Fig. 22.

Figure 3. Three-month-old Tg2576 mice show altered basic glutamatergic synaptic transmission and enhanced LTD.

a, Representative whole-cell traces recorded at $-65 \mathrm{mV}$ and $+20 \mathrm{mV}$ holding potential for wild-type (WT) and Tg2576 (Tg) mice (open circles indicate the time points at which the AMPA (peak) and the NMDA component were evaluated); and averaged AMPA/NMDA ratio in wild-type ( $n=8$ cells) and $\operatorname{Tg} 2576(n=9)$ mice. Data are represented as mean \pm s.e.m. $* P=0.037$. b, Representative traces (inter-stimulus interval: $100 \mathrm{~ms}$ ) and averaged PPR in wild-type ( $n=8$ cells) and Tg2576 ( $n=9)$ mice. c, Representative traces for wild-type and $\operatorname{Tg} 2576$ mice displaying spontaneous AMPAR-mediated miniature EPSCs (mEPSCs). d, Average amplitude and frequency of AMPAR mEPSC for wildtype $(n=16)$ and $\operatorname{Tg} 2576(n=15)$ mice. Data are represented as mean \pm s.e.m. $* P=0.044$. e, Representative traces of field excitatory post-synaptic potentials (fEPSPs) immediately before (black traces) and 50 min after (gray traces) high frequency stimulation (HFS). Averages of 10 traces for each example are shown. Bottom panel, time course of relative changes of fEPSP slopes for wild-type ( $n=20$ slices) and $\operatorname{Tg} 2576(n=14)$ mice. HFS was delivered at time $0 . \mathbf{f}$, Representative traces of fEPSPs before (black traces) and 50 min after (gray traces) low frequency stimulation (LFS). Average of 10 traces is shown for each example. Bottom panel, time course of relative changes of fEPSP slopes for wild-type ( $n=11$ slices) and Tg2576 $(n=12)$ mice. LFS was delivered at time $0 . * P=0.019$.

Figure 4. Tg2576 hippocampal dendritic spines accumulate active caspase-3 in the postsynaptic compartment and show apoptotic features.

a, Caspase-3 activity was revealed by a fluorimetric assay in both total hippocampal homogenate (Tot) and hippocampal synaptosomes (Syn) from wild-type (WT) and Tg2576 (Tg) mice. The 
histogram shows the caspase- 3 activity mean \pm s.d. (wild-type is indicated as $100 \%$ ). ${ }^{* * P} P=0.003$ (Tot), $* * P=0.002$ (Syn) ( $N=6$ mice for each age and genotype). b, Representative immunoblot showing presence of cleaved caspase-3 in hippocampal synaptosome (Syn) preparation, but not in total hippocampal homogenates (Tot). Ctrl: positive control for caspase-3 cleavage (proneural cells treated with staurosporine). Graph reports densitometric quantification of changes in grey values \pm s.d. $* * P=0.005(N=6)$. For full-length blots see Supplementary Fig. 22. c, Arrows indicate cleaved caspase-3-immunopositive post-synaptic compartments in CA1 dendritic spines. Arrowheads indicate cleaved caspase-3-immunonegative dendritic spines. Scale bar, $0.5 \mu \mathrm{m} . \mathrm{m}$ : mitochondrion; v: neurotransmitter vesicles. Graph shows the proportion of cleaved caspase-3-immunopositive dendritic spines to the total number found in all fields examined. ${ }^{*} P=0.018$ ( $N=3$ mice from each genotype, $n=100$ spines from each genotype). d, Quadrant analysis from flow cytometry apoptosis assay on synaptosomes. Representative density plot showing calcein AM fluorescence vs. annexin V fluorescence for large synaptosomal particles. Mean values \pm s.d: wild-type $22.3 \pm 6.8 \%, \operatorname{Tg} 2576$ $43.4 \pm 8.2 \%$. $* P=0.021$ ( $N=6$ mice from each genotype, $n=3$ independent experiments). e, Forward scatter signal/propidium iodide fluorescence analysis of hippocampal nuclei. Representative density plot showing propidium iodide (PI) incorporation in wild-type and Tg2576 nuclei preparation. Mean values \pm s.d.: wild-type $5.9 \pm 1.7 \%, \operatorname{Tg} 25765.3 \pm 1.5 \%$. $(N=6, n=3)$.

Figure 5. Inhibition of caspase-3 activity reduces GluR1 dephosphorylation, its removal from PSD and rescues glutamatergic synaptic transmission in Tg2576 mice.

a, Caspase-3 activity in Tg2576 hippocampal slices incubated with z-DEVD-fmk or vehicle control; data are expressed as mean \pm s.d. (control is indicated as $100 \%) . * P=0.026(2.5 \mu \mathrm{M}$ z-DEVD-fmk), $* P=0.019(5 \mu \mathrm{M} z$-DEVD- $f m k)(N=4$ mice for each experimental condition). $\mathbf{b}$, PSD proteins from z-DEVD-fmk or vehicle-treated slices were immunoblotted. The densitometric data are expressed as mean \pm s.d. (control is indicated as $100 \%$ ). ${ }^{*} P=0.033$ (GluR1; $2.5 \mu \mathrm{M}$ z-DEVD-fmk), ${ }^{*} P=0.031$ (GluR1pSer845; $2.5 \mu \mathrm{M}$ z-DEVD-fmk), ${ }^{*} P=0.028$ (GluR1; $5 \mu \mathrm{M} \quad$ z-DEVD-fmk), $* P=0.026$ 
(GluR1pSer845; $2.5 \mu \mathrm{M}$ z-DEVD-fmk) $(N=4)$. c, Linear regression on the caspase-3 activity ratio (z-DEVD-fmk/vehicle control) and immunoreactivity measures of GluR1 ratio (z-DEVD$\mathrm{fmk} / \mathrm{vehicle}$ control). Each point represents a single experiment ( $N=8$ mice). Correlation coefficient: -0.97 (Pearson correlation test; $* P=0.002$ ). d, Representative immunoblots of GluR1 and GluR1pSer845 in total protein extract from slices incubated with $5 \mu \mathrm{M}$ z-DEVD-fmk or with vehicle. The densitometric data are expressed as mean \pm s.d. (control is indicated as 100\%). $* P=0.028(N=4)$. e, Representative whole-cell traces recorded at $-65 \mathrm{mV}$ and $+20 \mathrm{mV}$ holding potential; and averaged ratio from neurons of $\mathrm{Tg}$ vehicle-treated ( $n=9$ cells), Tg z-DEVD-fmktreated (5 $\mu \mathrm{M}$ z-DEVD-fmk; $n=8)$, and WT untreated $(n=8)$ slices. The mean \pm s.e.m is shown. $* P=0.015 . \mathbf{f}$, Representative traces (inter-stimulus interval: $100 \mathrm{~ms}$ ) and average PPR from neurons of Tg vehicle-treated ( $n=9)$, Tg z-DEVD-fmk-treated (5 $\mu \mathrm{M}$ z-DEVD-fmk; $n=8)$ and WT untreated $(n=8)$ slices. For full-length blots see Supplementary Fig. 23.

\section{Figure 6. Inhibition of caspase-3 activity reduces calcineurin cleavage and activity.}

a, Representative immunoblot and densitometric analysis of $\mathrm{CN}-\mathrm{A}$ full length and its $\mathrm{N}$-terminal fragment in total protein extract from Tg2576 slices incubated with $5 \mu \mathrm{M}$ z-DEVD-fmk or with vehicle for 2 hrs. The data shown in the graph are expressed as mean \pm s.d. (control is indicated as $100 \%) . * P=0.039(N=4) . \mathbf{b}$, The slices were analyzed for calcineurin activity by monitoring ${ }^{32} \mathrm{P}$ release from radiolabeled purified RII substrate. As control of calcineurin activity, FK506 was added to protein extracts ( + FK506). The data are expressed as mean \pm s.d. (control is indicated as $100 \%) . * P=0.030(N=6)$.

Figure 7. Caspase-3 inhibition in vivo influences GluR1 distribution and rescues spine head size and memory function in Tg2576 mice.

a, $15 \mathrm{hrs}$ after intra-hippocampal injection of z-DEVD-fmk or vehicle into 3-month-old Tg2576 mice, caspase- 3 and calcineurin activities were analyzed. The data are expressed as mean \pm s.d. 
(control is indicated as $100 \%) . * P=0.012$ (caspase-3), $* P=0.020$ (calcineurin) $(N=5$ mice). b, Representative immunoblot and densitometric analysis of $\mathrm{CN}-\mathrm{A}$ full length and its $\mathrm{N}$-terminal fragment in total protein extract from Tg2576 hippocampus 15 hrs after z-DEVD-fmk or vehicle injection. The data shown in the graph are expressed as mean \pm s.d. (control is indicated as $100 \%$ ). * $P=0.031(N=4) . \mathbf{c}$, Representative immunoblots of hippocampus synaptic fractionation performed $15 \mathrm{hrs}$ after drug or vehicle injection and GluR1 densitometric quantification of changes in grey values expressed as mean \pm s.d. (control is indicated as $100 \%) \cdot{ }^{*}=0.034(N=4)$. TxP, PSDenriched fraction; P3, microsome-enriched fraction. For full-length blots see Supplementary Fig. 23. d, Left, representative photomicrographs of Golgi-stained apical dendrite segments after injection of z-DEVD-fmk or vehicle. Scale bar, $10 \mu \mathrm{m}$. Right, high-magnification micrograph of the area delineated by the box. Scale bar, $1 \mu \mathrm{m}$. Graph shows cumulative frequencies of spine head diameters. $P=0.002$ ( $N=4$ mice, $n=1000$ spine per group). e, Percentage of freezing time during CFC test in vehicle- and z-DEVD-fmk-treated wild-type (WT) and Tg2576 (Tg) mice. Data are expressed as mean \pm s.e.m. ${ }^{*} P=0.035$ (wild-type vehicle vs wild-type $\mathrm{z}$-DEVD- $f m k$ ), ${ }^{*} P=0.036$ (Tg2576 vehicle vs Tg2576 z-DEVD-fmk), ${ }^{* * *} P=0.0003$ ( $N=7$ mice for each experimental group). 


\section{Methods}

\section{Mice}

Heterozygous male $\operatorname{Tg} 2576$ mice $^{11}$ and wild-type littermates were used for all studies. All experiments were performed on independent groups of mice. Experiments were carried out in accordance with the European Community's Council Directive 86/609/EEC. Formal approval of the described experiments was obtained by the Italian Ministry of Health (D.L.vo 116/92).

\section{Contextual fear conditioning (CFC)}

After four days of handling each mouse was placed in the conditioning chamber (transparent Plexiglass cage, $28 \times 28 \times 10 \mathrm{~cm}$ ) for $120 \mathrm{~s}$ of free exploration period, followed by a series of 5 non signaled footshocks $(0.7 \mathrm{~mA}, 2 \mathrm{~s}$ duration, separated by $60 \mathrm{~s}$ intervals $)$ delivered through the grid floor. CFC was assessed $24 \mathrm{hrs}$ later by returning mice for $5 \mathrm{~min}$ to the conditioning chamber where no footshock was delivered. Fear memory was estimated by recording the time spent in freezing behavior, defined as the total absence of all but respiratory movements associated with a crouching posture. Mice behavior was videotaped by means of a video camera connected to a computer equipped with a dedicated software (Noldus Ethovision System, The Netherlands). Motor activity during the free exploration period was automatically scored by means of Noldus Ethovision system.

\section{Golgi Cox staining and dendritic spines measurements}

Naïve male mice were intracardially perfused with $0.9 \%$ saline solution. The brains were impregnated by immersion in a standard Golgi-Cox solution (1\% potassium dichromate, $1 \%$ mercuric chloride, $0.8 \%$ potassium chromate) for 6 days, transferred to a $30 \%$ sucrose solution for 2 days, and then sectioned coronally $(100 \mu \mathrm{m})$. Sections were mounted on gelatinized slides and stained according to the Gibb and Kolb method. Fully impregnated pyramidal neurons laying in the CA1 region of the dorsal hippocampus were identified under low magnification (20x/0.5NA). In each brain, 11 neurons displaying dendritic tree without obvious truncations and balanced per 
hemisphere were analyzed under higher magnification (63x/0.75NA). Measurements were carried out using a microscope (DMLB, Leica) equipped with a camera (resolution 2600x2600, Axiocam, Zeiss), and the KS300 3.0 system (Zeiss). A computer-based neuron tracing system (Neurolucida, Microbrightfield) was used to trace single neurons. Spine density was assessed by counting the number of spines in five $20 \mu \mathrm{m}$ dendrite segments per neuron chosen in branch orders 2-4 of apical and basal dendrites. All protrusions, with or without bulbous expansion but no longer than $2 \mu \mathrm{m}$, were counted as spines if they were in continuity with the dendritic shaft. The average spine density (number of spines per $1 \mu \mathrm{m}$ length segment) was averaged for a neuron mean. To determine dendritic spine morphology, the spines on five $20 \mu \mathrm{m}$ apical dendrite segments per neuron were acquired under higher magnification (100x/1.25NA) using a digital camera (Axiocam, Zeiss). Morphology was estimated by measuring spine head diameter by means of the public domain ImageJ software (HYPERLINK “http://.info.nih.gov/ij/”). Values were averaged for neuron and cumulative frequencies compared among groups. Total dendrite length was automatically calculated by the Neurolucida software after each neuron was traced.

\section{In vitro slice preparation and electrophysiology}

Hippocampal slices were prepared as described previously ${ }^{48}$. Slices were recorded at $32 \pm 1^{\circ} \mathrm{C}$ in oxygenated artificial cerebrospinal fluid (aCSF) containing $126 \mathrm{mM} \mathrm{NaCl}, 26 \mathrm{mM} \mathrm{NaHCO} 3,2.5$ $\mathrm{mM} \mathrm{KCl}, 1.25 \mathrm{mM} \mathrm{NaH}_{2} \mathrm{PO}_{4}, 2 \mathrm{mM} \mathrm{MgSO}_{4}, 2 \mathrm{mM} \mathrm{CaCl}_{2}, 10 \mathrm{mM}$ glucose, gassed with 95\% $\mathrm{O}_{2}$ / $5 \% \mathrm{CO}_{2}, \mathrm{pH} 7.4$, in the continuous presence of $100 \mu \mathrm{M}$ picrotoxin. Field excitatory post-synaptic potentials (fEPSPs) were recorded in the stratum radiatum of the CA1 and stimuli delivered by a monopolar electrode. Input/output relationships were generated by calculating the fEPSP initial slope (to avoid population spike contamination) corresponding to a given fiber volley amplitude (100 to $200 \mathrm{mV}$ in increments of $50 \mathrm{mV}$ ). For LTP experiments, fEPSPs were stimulated at $0.1 \mathrm{~Hz}$ and LTP was induced by 1 train of 100 stimuli at $100 \mathrm{~Hz}$. For LTD, slices were superfused with 
aCSF containing $\left[\mathrm{Ca}^{2+}\right]=4 \mathrm{mM}$ and LTD was induced with a low frequency stimulation (LFS) protocol of 900 pulses at $1 \mathrm{~Hz}$.

Whole cell patch clamp recordings were obtained from CA1 pyramidal cells as described previously $^{48}$. Recordings were made using intracellular solution $\left(117.5 \mathrm{mM} \mathrm{CsMeSO}_{4}, 15.5 \mathrm{mM}\right.$, CsCl, $10 \mathrm{mM}$ TEACl, $8 \mathrm{mM} \mathrm{NaCl}, 1.25 \mathrm{mM} \mathrm{NaH}_{2} \mathrm{PO}_{4}, 10 \mathrm{mM}$ HEPES, $0.25 \mathrm{mM}$ EGTA, $4 \mathrm{mM}$ MgATP, $0.3 \mathrm{mM}$ NaGTP, $\mathrm{pH}=7.3$, osmolarity $290 \mathrm{mOsm})$. Series (10-20 M $\Omega$ ) and input (100-200 $\mathrm{M} \Omega$ ) resistances were monitored online throughout each experiment. For the AMPAR/NMDAR ratio, cells were held at $-65 \mathrm{mV}$ to record AMPAR mediated currents and at $+20 \mathrm{mV}$, to record AMPAR+NMDAR mediated currents. The AMPAR excitatory post-synaptic current (EPSC) amplitude was calculated at the peak (averaged over a $1 \mathrm{~ms}$ window) and the NMDA EPSC amplitude was calculated by measuring the current $(2 \mathrm{~ms}$ window $)$ at $60 \mathrm{~ms}$ after stimulation artifact. For paired pulse ratios (PPR) measurements, two pulses were delivered $100 \mathrm{~ms}$ apart at -65 $\mathrm{mV}$. Spontaneous AMPAR-mediated miniature EPSCs (mEPSCs) were recorded at $-65 \mathrm{mV}$ holding potential in $\left[\mathrm{Ca}^{2+}\right]$ out $=4 \mathrm{mM}$ and $\left[\mathrm{K}^{+}\right]$out $=7 \mathrm{mM}$, in the presence of $0,5 \mu \mathrm{M}$ tetrodoxin (Latoxan) and $100 \mu \mathrm{M}$ picrotoxin (Sigma-Aldrich). Spontaneous events were analysed using using the Clampfit software (Molecular Devices) through a template search with variable amplitude.

For experiments in Fig. 5, slices were incubated for 2 hrs with $5 \mu \mathrm{M}$ z-DEVD-fmk at RT, and then transferred to the recording chamber. Only cells successfully recorded within $1 \mathrm{hr}$ of the end of incubation were included for analysis.

\section{Surgical procedures}

Mice were anesthetized with chloral hydrate $(400 \mathrm{mg} / \mathrm{kg})$ and mounted on stereotaxic apparatus. Bilateral injecting cannulae were inserted in the hippocampus (coordinates relative to bregma: anteroposterior $-2 \mathrm{~mm}$, lateral $\pm 1.7 \mathrm{~mm}$, ventral $1.9 \mathrm{~mm}$ ). Injections were performed using a $10 \mu \mathrm{l}$ Hamilton syringe connected to a gauge injector. 
One group of mice was injected in one hemi-hippocampus with $1 \mu \mathrm{l}$ of $174 \mu \mathrm{M}$ z-DEVD-fmkDMSO solution (Imgenex) diluted in aCSF. As control, $1 \mu \mathrm{l}$ of aCSF-DMSO solution was injected in the controlateral hemi-hippocampus. A separate group of mice was injected with $1 \mu \mathrm{l}$ of $3.5 \mathrm{mM}$ $\gamma$-secretase inhibitor IX (DAPT, Calbiochem) in one hemi-hippocampus and $1 \mu 1$ of DMSO in the controlateral hemi-hippocampus. Another group of mice was injected with $1 \mu$ of $0.7 \mathrm{mM}$ apoptosome inhibitor (QM56, kindly provided by Dr. Pérez-Payá) diluted in PBS. As control, $1 \mu 1$ of PBS was injected in the controlateral hemi-hippocampus. Injections of inhibitor or vehicle were balanced for hemispheres. Mice were sacrificed 15 hrs (z-DEVD-fmk- and DAPT-injected mice) and $48 \mathrm{hrs}$ (QM56-injected mice) after the injections for brains collection. Two independent groups of naïve mice were injected bilaterally in the hippocampus with either z-DEVD-fmk or DAPT 15 hrs before training in CFC task. Relative controls were injected respectively with vehicle solutions.

\section{Post-synaptic density (PSD) preparation}

The brain was rapidly dissected and the hippocampus was homogenized in homogenization buffer (320 mM sucrose, $1 \mathrm{mM}$ HEPES pH 7.4, $1 \mathrm{mM} \mathrm{MgCl}_{2}, 1 \mathrm{mM}$ EDTA, $1 \mathrm{mM} \mathrm{NaHCO}, 1 \mathrm{mM}$ PMSF, $1 \mathrm{mM} \mathrm{Na} \mathrm{VO}_{4}, 20 \mathrm{mM} \beta$-glycerophosphate, 5mM NaF, with protease inhibitors - Sigma) with 10 strokes of a tight-fitting glass Dounce tissue grinder $(7 \mathrm{ml}$; Wheaton). The homogenate was centrifuged at $1,000 \times g$ for $10 \mathrm{~min}$ and the resulting supernatant was centrifuged at $3,000 \times g$ for 15 min. The pellet was resuspended in $1 \mathrm{mM}$ HEPES $\mathrm{pH} 7.4$ containing protease inhibitors and centrifuged at $100,000 \times g$ for $1 \mathrm{hr}$. The resulting pellet was resuspended in a buffer containing 75 $\mathrm{mM} \mathrm{KCl}, 1 \%$ Triton $\mathrm{X}-100$ and proteases inhibitors and centrifuged at $100,000 \times \mathrm{g}$ for $1 \mathrm{hr}$. The supernatant was referred as Triton X-100 Soluble Fraction (TSF). The pellet containing the PSD fraction (Triton X-100 Insoluble Fraction, TIF) was resuspended in RIPA buffer (50 mM Tris-HCl $\mathrm{pH} 7.4,1 \%$ Triton X-100, 0.25\% Na-deoxycholate, $150 \mathrm{mM} \mathrm{NaCl}, 5 \mathrm{mM} \mathrm{MgCl} 2,1 \mathrm{mM}$ EDTA, $0,1 \%$ SDS, protease inhibitors), sonicated and incubated on ice for $20 \mathrm{~min}$. The samples were 
centrifuged at $11,500 \times g$ for $10 \mathrm{~min}$ and the protein concentration of resulting supernatant was determined.

\section{Sub-synaptic fractionation}

Hippocampus was homogenized in homogenization buffer $(320 \mathrm{mM}$ sucrose, $10 \mathrm{mM}$ Tris- $\mathrm{HCl} \mathrm{pH}$ 7.4, $1 \mathrm{mM}$ EDTA, $1 \mathrm{mM} \mathrm{NaHCO}$, $1 \mathrm{mM}$ PMSF, $1 \mathrm{mM} \mathrm{Na}_{3} \mathrm{VO}_{4}, 5 \mathrm{mM} \mathrm{NaF}$, with protease inhibitors) with 10 strokes of a tight-fitting glass Dounce tissue grinder. The homogenate was centrifuged at $1,000 \times g$ for $10 \mathrm{~min}$ and the resulting supernatant was centrifuged at $10,000 \times g$ for 15 min. Both the pellet (P2) and the supernatant (S2) were stored. The P2 was resuspended in homogenization buffer, incubated $40 \mathrm{~min}$ on ice and centrifuged at $32,000 \times g$ for $20 \mathrm{~min}$. The resulting pellet $(\mathrm{TxP})$ containing PSD was processed for protein extraction as described for "PSD preparation". The S2 was centrifuged at $100,000 \times g$ for $1 \mathrm{hr}$ and the microsomal-enriched proteins were extracted from resulting pellet (P3).

\section{Synaptosome preparation}

Hippocampus was homogenized in homogenization buffer (320 mM sucrose, 4 mM Hepes pH 7.4, $1 \mathrm{mM}$ EGTA, $1 \mathrm{mM}$ PMSF, with protease inhibitors) with 10 strokes of a tight-fitting glass Dounce tissue grinder. The homogenate was centrifuged at $1,000 \times g$ for $10 \mathrm{~min}$ and the resulting supernatant was centrifuged at $12,000 \times g$ for $15 \mathrm{~min}$. The pellet was resuspended in homogenization buffer and centrifuged at $13,000 \times g$ for 15 min to obtain the final pellet containing synaptosome-enriched fraction.

\section{Nuclei isolation}

Nuclei enriched fraction was prepared by the same hippocampal homogenate used for synaptosomes preparation. The homogenate was centrifuged at $800 \times g$ for $5 \mathrm{~min}$, the pellet was washed twice with cold PBS, resuspended in hypotonic lysis buffer $(10 \mathrm{mM}$ Tris- $\mathrm{HCl} \mathrm{pH} 7.4,0.1$ 
M sucrose, $10 \mathrm{mM} \mathrm{NaCl}, 1 \mathrm{mM}$ DTT, $1 \mathrm{mM}$ PMSF, with protease inhibitors), vortexed for $15 \mathrm{~s}$ and incubated on ice for $15 \mathrm{~min}$. Then, 1.8 M sucrose was added, samples were mixed and loaded on top of a 1.1 M sucrose cushion and centrifuged at $14,700 \times g$ for $25 \mathrm{~min}$ in a SW60Ti rotor. The pellet was resuspended in PBS and centrifuged at $1000 \times g$ for 5 min to obtain the pellet containing nuclei.

\section{Total homogenate preparation from hippocampal tissue}

Hippocampal tissue was homogenized in lysis buffer $(320 \mathrm{mM}$ sucrose, $50 \mathrm{mM} \mathrm{NaCl}, 50 \mathrm{mM}$ Tris$\mathrm{HCl} \mathrm{pH} 7.5,1 \%$ Triton X-100, $0.5 \mathrm{mM}$ Na-orthovanadate, $5 \mathrm{mM} \beta$-glycerophosphate, with proteases inhibitors), incubated on ice for $30 \mathrm{~min}$ and centrifuged at $13,000 \times g$ for $10 \mathrm{~min}$. The total protein content of resulting supernatant was determined.

\section{Fluorimetric assay of caspase-3 activity}

Synaptosomes-containing pellet, total hippocampal tissue and hippocampal slices were homogenized in lysis assay buffer (100 mM Hepes pH 7.4, 0.1\% Chaps, 1 mM EDTA, 10 mM DTT, $1 \mathrm{mM}$ PMSF) and lysed by freezing in liquid $\mathrm{N}_{2}$ and thawing at $37^{\circ} \mathrm{C}$ three times. After centrifugation at $11,500 \times g$ for 5 min the protein concentration of resulting supernatant was determined and the same amount of proteins was incubated at $37^{\circ} \mathrm{C}$ in lysis assay buffer containing $50 \mu \mathrm{M}$ Ac-DEVD-AMC (Calbiochem). The fluorescence was measured with $380 \mathrm{~nm}$ excitation wavelength and $460 \mathrm{~nm}$ emission wavelength.

\section{Immunoelectron microscopy}

Animals were intracardially perfused with a calcium-free Ringer's variant, followed by fixative solution (4\% depolymerized paraformaldehyde in $0.12 \mathrm{M}$ sodium phosphate buffer, $\mathrm{pH}$ 7.2). Brains were sagittally cut to obtain $80 \mu \mathrm{m}$ thick sections. The free-floating sections were incubated with an anti-cleaved caspase-3 antibody (1:100; Cell Signaling). Then, sections were post-fixed with 1\% 
$\mathrm{OsO}_{4}$, dehydrated, infiltrated in epoxy resin and flat-embedded in the same medium. Hippocampal CA1 fields were re-embedded on epon blanks and further cut by a Reichert Ultracut S ultramicrotome (Leica Microsystems), to obtain ultrathin (70-80 nm) sections. These were collected on nickel grids, lightly contrasted with uranyl acetate and lead citrate and finally observed under CM120 electron microscope (Philips), equipped with a videocamera. Digitized images were electronically captured and composed in Adobe Photoshop CS3. To quantitatively evaluate the occurrence of caspase-3-containing spines in the different genetic conditions, we analyzed DAB stained ultrathin sections.

\section{Flow Cytometry}

Synaptosome flow cytometry was performed as previously described ${ }^{49}$. Synaptosomes-containing pellet was resuspended in binding buffer (10 mM Hepes, $\mathrm{pH} 7.4,150 \mathrm{mM} \mathrm{NaCl}, 5 \mathrm{mM} \mathrm{KCl}, 5 \mathrm{mM}$ $\mathrm{MgCl}_{2}, 1.8 \mathrm{mM} \mathrm{CaCl}_{2}$ ), $100 \mu \mathrm{l}$ aliquots were incubated $10 \mathrm{~min}$ at RT with dye solution and then diluted in PBS (final volume $500 \mu$ ) for immediate flow cytometry analysis. Final dye concentration was $100 \mathrm{nM}$ for calcein AM (Molecular Probes) and $1 \mathrm{mg} / \mathrm{ml}$ for PE-annexin V (BD Pharmingen). The samples were acquired on a FACS Canto flow cytometer. Polystyrene beads $(0.75,1.4$ and $4.5 \mu \mathrm{m})$ were run on the instrument to standardize forward scatter measurements.

Nuclei-containing pellet was resuspended in PBS, and propidium iodide, PI (Sigma), solution was added to give a final concentration of $1 \mathrm{mg} / \mathrm{ml}$. The samples were immediately analyzed with appropriate software (FACS Canto and FACS Diva; Becton Dickson). Scatter profiles for fluorescence intensities FL-2 (PI) were plotted. The percentage of cells in each quadrant was determined with Flowjo version 8.6.1.

\section{Hippocampal slices treatment}

Coronal brain slices ${ }^{48}$ were cut along a sagittal plane into 2 hemisections. The homolateral sections were incubated for $2 \mathrm{hrs}$ at RT in aCSF (gassed with $95 \% \mathrm{O}_{2} / 5 \% \mathrm{CO}_{2}, \mathrm{pH} 7.4$ ) containing 2.5 or 5 
$\mu \mathrm{M}$ z-DEVD-fmk (Imgenex), $5 \mu \mathrm{M}$ z-FA-fmk (Imgenex) or $10 \mu \mathrm{M}$ FK506 (Sigma), and the controlateral sections in aCSF containing DMSO.

\section{Cathepsin B activity assay}

Hippocampal slices were homogenized in lysis buffer $(20 \mathrm{mM}$ Hepes pH 7.4, $10 \mathrm{mM} \mathrm{KCl,} 1.5 \mathrm{mM}$ $\mathrm{MgCl}_{2}, 0.5 \% \mathrm{NP} 40,1 \mathrm{mM}$ DTT, $1 \mathrm{mM}$ PMSF), sonicated for $10 \mathrm{~s}$ and centrifuged at $15,000 \times \mathrm{g}$ for $30 \mathrm{~min}$. The protein concentration of resulting supernatant was determined, the same amount of proteins was incubated at $37^{\circ} \mathrm{C}$ in assay buffer $(50 \mathrm{mM}$ Na-acetate $\mathrm{pH} 6.0,4 \mathrm{mM}$ EDTA, $8 \mathrm{mM}$ DTT) containing $50 \mu \mathrm{M}$ z-RR-AMC (Biomol International) and the fluorescence was measured with $380 \mathrm{~nm}$ excitation wavelength and $460 \mathrm{~nm}$ emission wavelength.

\section{Calcineurin activity assay}

CaN activity was assayed by monitoring ${ }^{32} \mathrm{P}$ release from radiolabeled purified RII substrate peptide $^{50}$. The hippocampal tissues were homogenized in buffer A (50 mM Tris- $\mathrm{HCl} \mathrm{pH} 7.4,1 \mathrm{mM}$ EDTA, $1 \mathrm{mM} \mathrm{CaCl} 2,1 \mathrm{mM}$ PMSF, $1 \mathrm{mM} \mathrm{DTT}$ ), subjected to repeated freezing in liquid $\mathrm{N}_{2}$ and thawing at $37^{\circ} \mathrm{C}$, and centrifuged at $17,000 \times g$ for $10 \mathrm{~min} .10 \mu \mathrm{g}$ of total proteins from the clear supernatant was diluted with an equal volume of buffer B (100 mM Tris-HCl pH 7.4, $1 \mathrm{mM} \mathrm{MnCl}$, $0.1 \mathrm{mM} \mathrm{CaCl}_{2}, 1 \mathrm{mM} \mathrm{DTT}$ ) and immediately processed for enzyme assay. Addition of $1 \mathrm{mM}$ $\mathrm{NiSO}_{4}$ in buffer $\mathrm{B}$ was used to obtain maximal activation of the enzyme together with $200 \mathrm{nM}$ okadaic acid at a final concentration known to inhibit phosphatase activities other than calcineurin. Addition of $\mathrm{MgCl}_{2}$ was avoided to prevent activation of protein phosphatase 2C. 'Ca-free' conditions of calcineurin assay were obtained by omission of calcium in both A an B buffers. Addition of calcineurin inhibitor (10 $\mu \mathrm{M}$ FK506) to clear supernatant was used as control.

\section{Immunoblotting analysis and antibodies}


Proteins were applied to SDS-PAGE and electroblotted on a PVDF membrane. Immunoblotting analysis was performed using a chemiluminescence detection kit. The relative levels of immunoreactivity were determined by densitometry using the software ImageQuant 5.0. Antibodies to caspase-3, cleaved caspase-3, cleaved PARP, caspase-6 and caspase-7 were purchased from Cell Signaling, Apaf1 from Alexis Biochemicals, GluR1, GluR1pSer831 and NMDAc2 from Upstate,

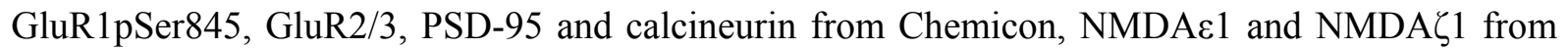
Santa Cruz, NSF from Synaptic Systems, aCaMKII from Zymed Laboratories, mGluR5 and synaptophysin from Abcam, Rab11 from BD Biosciences, calpain-1 from Calbiochem, actin and $\beta$ tubulin from Sigma.

\section{Coomassie staining}

PSD proteins were resolved by SDS-PAGE. After electrophoresis the gels were fixed for $30 \mathrm{~min}$ in cold fixing solution $\left(50 \%\right.$ MetOH, 10\% acetic acid, $\left.40 \% \mathrm{ddH}_{2} \mathrm{O}\right)$, stained with Coomassie solution ( $0.2 \%$ Coomassie blue, $20 \% \mathrm{MetOH}, 10 \%$ acetic acid) for $30 \mathrm{~min}$ and washed abundantly with cold destaining solution (45\% MetOH, 10\% acetic acid, 45\% dd $\mathrm{H}_{2} \mathrm{O}$ ).

\section{TUNEL assay}

TUNEL assay was performed on $10 \mu \mathrm{m}$ paraffin-embedded brain sections by using the ApopTag Fluorescein In Situ Apoptosis Detection Kit purchased from Chemicon. The assay was carried out according to manufacturer's instructions.

\section{Measurement of carbonylated proteins}

Carbonylated proteins were detected using the Oxyblot kit (Chemicon) according to manufacturer's instructions. Briefly, $5 \mu \mathrm{g}$ of hippocampal proteins were reacted with dinitrophenylhydrazine (DNP) solution for $15 \mathrm{~min}$ at $25^{\circ} \mathrm{C}$. The DNP-reacted proteins were resolved on $10 \%$ SDS-polyacrylamide gel and were identified by immunoblotting using an anti-DNP antibody. 


\section{Primary neuronal cell culture: preparation and analysis}

The primary neuronal cell cultures were prepared from cortical and hippocampal tissue of E15

embryos. Females Apaf1 $^{+/-44}$ were crossed with males Tg2576/Apaf1 ${ }^{+/}$to obtain transgenic and transgenic lacking of Apafl protein neurons.

Cortical and hippocampal tissues of embryos were dissociated in $0.025 \%$ Trypsin $\left(7 \mathrm{~min}\right.$ at $\left.37^{\circ} \mathrm{C}\right)$, dissociation was blocked with FBS and samples were centrifuged for $3 \mathrm{~min}$ at $220 \times g$. The tissue was resuspended in MEM\#1 medium (25 mM glucose, 1\% FBS, $2 \mathrm{mM}$ L-glutamine, $0.1 \mathrm{mg} / \mathrm{ml}$ gentamicin, MEM medium) and centrifuged $10 \mathrm{~min}$ at $220 \times \mathrm{g}$. The resulting cells pellet was resuspended in MEM\#2 medium (25 mM glucose, 5\% FBS, 5\% HS, $2 \mathrm{mM} \mathrm{L-glutamine,} 0.1 \mathrm{mg} / \mathrm{ml}$ gentamicin, MEM medium). Cells were plated in MEM\#2 medium and incubated in a humidified $37^{\circ} \mathrm{C}, 5 \% \mathrm{CO}_{2}$ incubator; after $1 \mathrm{hr}$ all medium were replaced with Neurobasal medium (2\% B-27 minus AO, $2 \mathrm{mM}$ L-glutamine, $0.1 \mathrm{mg} / \mathrm{ml}$ gentamicin, Neurobasal medium) and primary neurons were cultured for 7 days.

To obtain total lysates for immunoblotting analysis, neurons were scraped in RIPA buffer (50 mM Tris- $\mathrm{HCl} \mathrm{pH}$ 7.4, 1\% Triton X-100, 0.25\% Na-deoxycholate, $150 \mathrm{mM} \mathrm{NaCl,} 5 \mathrm{mM} \mathrm{MgCl} 2,1 \mathrm{mM}$ EDTA, $0.1 \%$ SDS, protease inhibitors), sonicated, incubated $20 \mathrm{~min}$ on ice and centrifuged at $11500 \times g$ for $10 \mathrm{~min}$. The protein content of resulting supernatant was determined.

\section{Data analysis and statistics}

Behavioral, total dendrite length, immunoblotting, immunohistochemistry, electrophysiology, flow cytometry and enzyme activity data analysis were performed with a two tailed Student's $t$ test.

Spine density data were analyzed by two-way ANOVA with age and genotype as independent variables, Behavioral data from mice treated with z-DEVD-fmk were analyzed by two-way ANOVA with treatment and genotype as independent variables. 
The Kolmogorov-Smirnov test was used to compare cumulative frequencies of spines heads diameters. In all cases $P<0.05$ was considered statistically significant. 


\section{References}

1. Arendt, T. Synaptic degeneration in Alzheimer's disease. Acta Neuropathol. 118, 167-179 (2009).

2. Morrison, J.H. \& Hof, P.R. Life and death of neurons in the aging brain. Science 278, 412-419 (1997).

3. Bookheimer, S.Y. et al. Patterns of brain activation in people at risk for Alzheimer's disease. $N$. Engl. J. Med. 343, 450-456 (2000).

4. Elias, M.F et al. The preclinical phase of Alzheimer disease: A 22-year prospective study of the Framingham Cohort. Arch. Neurol. 57, 808-813 (2000).

5. Zhou, Y. et al. Abnormal connectivity in the posterior cingulate and hippocampus in early Alzheimer's disease and mild cognitive impairment. Alzheimers Dement. 4, 265-270 (2008).

6. Scheff, S.W., Price, D.A., Schmitt, F.A., DeKosky, S.T., \& Mufson, E.J. Synaptic alterations in CA1 in mild Alzheimer disease and mild cognitive impairment. Neurology 68, 1501-1508 (2007).

7. Bourne, J.N \& Harris, K.M. Balancing structure and function at hippocampal dendritic spines. Annu. Rev. Neurosci. 31, 46-67 (2008).

8. Ferrer, I., Guionnet, N., Cruz-Sánchez, F., \& Tuñón T. Neuronal alterations in patients with dementia: a Golgi study on biopsy samples. Neurosci. Lett. 114, 11-16 (1990).

9. Lanz, T.A., Carter, D.B. \& Merchant, K.M. Dendritic spine loss in the hippocampus of young PDAPP and Tg2576 mice and its prevention by the ApoE2 genotype. Neurobiol. Dis. 13, 246$253(2003)$

10. Mattson, M.P., Keller, J.N., \& Begley, J.G. Evidence for synaptic apoptosis. Exp. Neurol. 153, 35-48 (1998).

11. Hsiao, K. et al. Correlative memory deficits, Abeta elevation, and amyloid plaques in transgenic mice. Science 274, 177-178 (1996). 
12. Jacobsen, J.S. et al. Early-onset behavioral and synaptic deficits in a mouse model of Alzheimer's disease. Proc. Natl. Acad. Sci. U.S.A. 103, 5161-5166 (2006).

13. Kawarabayashi, T. et al. Age-dependent changes in brain, CSF, and plasma amyloid (beta) protein in the Tg2576 transgenic mouse model of Alzheimer's disease. J. Neurosci. 21, 372-381 (2001).

14. Irizarry, M.C., McNamara, M., Fedorchak, K., Hsiao, K. \& Hyman, B.T. APPSw transgenic mice develop age-related A beta deposits and neuropil abnormalities, but no neuronal loss in CA1.J. Neuropathol. Exp. Neurol. 56, 965-973 (1997).

15. Westerman, M.A. et al. The relationship between Abeta and memory in the Tg2576 mouse model of Alzheimer's disease. J. Neurosci. 22, 1858-1867 (2002).

16. Janus, C. et al. A beta peptide immunization reduces behavioural impairment and plaques in a model of Alzheimer's disease. Nature 408, 979-982 (2000).

17. Chen, G. et al. A learning deficit related to age and beta-amyloid plaques in a mouse model of Alzheimer's disease. Nature 408, 975-979 (2000).

18. Phillips, R.G. \& LeDoux, J.E. Differential contribution of amygdala and hippocampus to cued and contextual fear conditioning. Behav. Neurosci. 106, 274-285 (1992).

19. Lee, I. \& Kesner, R.P. Differential contributions of dorsal hippocampal subregions to memory acquisition and retrieval in contextual fear-conditioning. Hippocampus 14, 301-310 (2004).

20. Lajtha, A., Perez-Polo, J.R. \& Rossner S. Handbook of Neurochemistry and Molecular Neurobiology (Springer US Press). (2008).

21. Li, S. et al. Soluble oligomers of amyloid Beta protein facilitate hippocampal long-term depression by disrupting neuronal glutamate uptake. Neuron 62, 788-801 (2009).

22. Almeida, C.G. et al. Beta-amyloid accumulation in APP mutant neurons reduces PSD-95 and GluR1 in synapses. Neurobiol Dis. 20, 187-198 (2005).

23. Malinow, R. \& Malenka, R.C. AMPA receptor trafficking and synaptic plasticity. Annu. Rev. Neurosci. 25, 103-126 (2002). 
24. Chapman, P.F. et al. Impaired synaptic plasticity and learning in aged amyloid precursor protein transgenic mice. Nat. Neurosci. 2, 271-276 (1999).

25. Lee, H.K., Kameyama, K., Huganir, R.L. \& Bear, M.F. NMDA induces long-term synaptic depression and dephosphorylation of the GluR1 subunit of AMPA receptors in hippocampus. Neuron 21, 1151-1162 (1998).

26. van Engeland, M., Nieland, L.J., Ramaekers, F.C., Schutte, B., \& Reutelingsperger, C.P. Annexin V-affinity assay: a review on an apoptosis detection system based on phosphatidylserine exposure. Cytometry 31, 1-9 (1998).

27. Green, D.R. \& Kroemer, G. The pathophysiology of mitochondrial cell death. Science 305, 626-629 (2004).

28. Schulze-Osthoff, K., Ferrari, D., Los, M., Wesselborg, S. \& Peter, M.E. Apoptosis signaling by death receptors. Eur. J. Biochem. 254, 439-459 (1998).

29. Hsieh, H. et al. AMPAR removal underlies Abeta-induced synaptic depression and dendritic spine loss. Neuron 52, 831-843 (2006).

30. Mukerjee, N., McGinnis, K.M., Park, Y.H., Gnegy, M.E. \& Wang, K.K. Caspase-mediated proteolytic activation of calcineurin in thapsigargin-mediated apoptosis in SH-SY5Y neuroblastoma cells. Arch. Biochem. Biophys. 379, 337-343 (2000).

31. Vicent, M.J. \& Pérez-Payá, E. Poly-L-glutamic acid (PGA) aided inhibitors of apoptotic protease activating factor 1 (Apaf-1): an antiapoptotic polymeric nanomedicine. J Med. Chem. 49, 3763-3765 (2006).

32. Santamaría, B., et al. A nanoconjugate Apaf-1 inhibitor protects mesothelial cells from cytokine-induced injury. PLoS One. 4, e6634 (2009).

33. Salvesen, G.S. \& Dixit, V.M. Caspases: intracellular signaling by proteolysis. Cell 91, 443-446 (1997).

34. Bravarenko, N.I. et al. Caspase-like activity is essential for long-term synaptic plasticity in the terrestrial snail Helix. Eur. .J Neurosci. 23, 129-140 (2006). 
35. Huesmann, G.R. \& Clayton, D.F. Dynamic role of postsynaptic caspase-3 and BIRC4 in zebra finch song-response habituation. Neuron 52, 1061-1072 (2006).

36. Li, Z. et al. Caspase-3 activation via mitochondria is required for long-term depression and AMPA receptor internalization. Cell 141, 859-871 (2010).

37. Marín, N. et al. Beta-amyloid-induced activation of caspase-3 in primary cultures of rat neurons. Mech. Ageing Dev. 119, 63-67 (2000).

38. Gervais, F.G. et al. Involvement of caspases in proteolytic cleavage of Alzheimer's amyloidbeta precursor protein and amyloidogenic A beta peptide formation. Cell 97, 395-406 (1999).

39. Stadelmann, C. et al. Activation of caspase-3 in single neurons and autophagic granules of granulovacuolar degeneration in Alzheimer's disease. Evidence for apoptotic cell death. Am. J. Pathol. 155, 1459-1466 (1999).

40. Louneva, N. et al. Caspase-3 is enriched in postsynaptic densities and increased in Alzheimer's disease. Am. J. Pathol. 173, 1488-1495 (2008).

41. Kuida, K. et al. Decreased apoptosis in the brain and premature lethality in CPP32-deficient mice. Nature 384, 368-372 (1996).

42. Hakem, R. et al. Differential requirement for caspase 9 in apoptotic pathways in vivo. Cell 94, 339-352 (1998).

43. Kuida, K. et al. Reduced apoptosis and cytochrome c-mediated caspase activation in mice lacking caspase 9. Cell 94, 325-337 (1998).

44. Cecconi, F., Alvarez-Bolado, G., Meyer, B.I., Roth, K.A. \& Gruss, P. Apaf1 (CED-4 homolog) regulates programmed cell death in mammalian development. Cell 94, 727-737 (1998).

45. Takahashi, R.H. et al.. Oligomerization of Alzheimer's beta-amyloid within processes and synapses of cultured neurons and brain. J. Neurosci. 24, 3592-3599 (2004).

46. Manczak, M. et al. Mitochondria are a direct site of A beta accumulation in Alzheimer's disease neurons: implications for free radical generation and oxidative damage in disease progression. Hum. Mol. Genet. 15, 1437-1449 (2006). 
47. Cho, D.H.et al. S-nytrosylation of Drp1 mediates beta-amyloid-related mitochondrial fission and neuronal injury. Science 324, 102-105 (2009).

48. Marie, H., Morishita, W., Yu, X., Calakos, N. \& Malenka, R.C. Generation of silent synapses by acute in vivo expression of CaMKIV and CREB. Neuron 45, 741-752 (2005).

49. Gylys, K.H., Fein, J.A., Wiley, D.J. \& Cole, G.M. Rapid annexin-V labeling in synaptosomes. Neurochem. Int. 44, 125-131 (2004).

50. Ferri, A. et al. Calcineurin activity is regulated both by redox compounds and by mutant familial amyotrophic lateral sclerosis-superoxide dismutase. J. Neurochem. 75, 606-613 (2000). 
D'Amelio et al., Figure 1
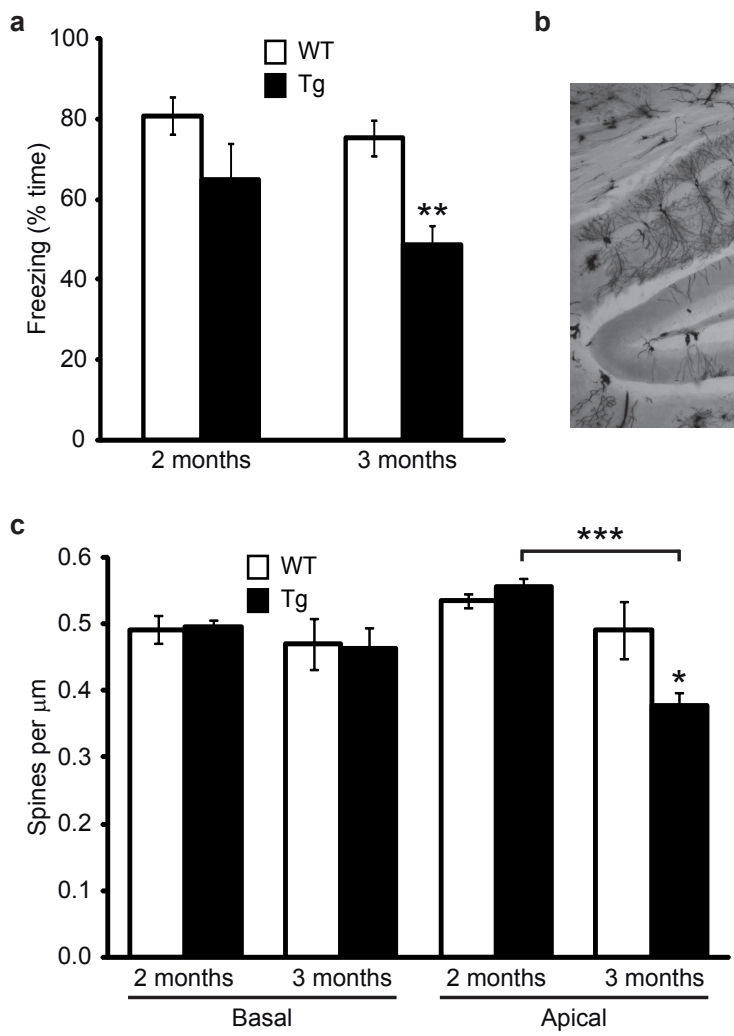

b

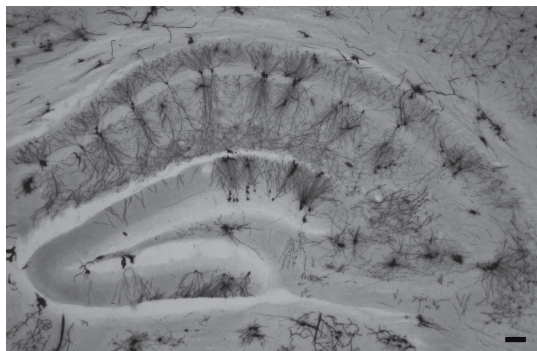

d

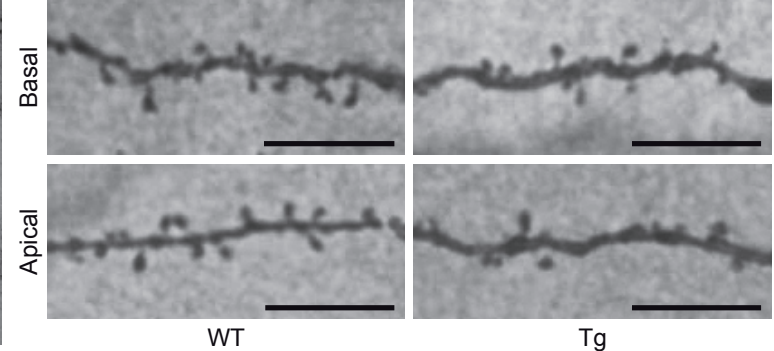

e

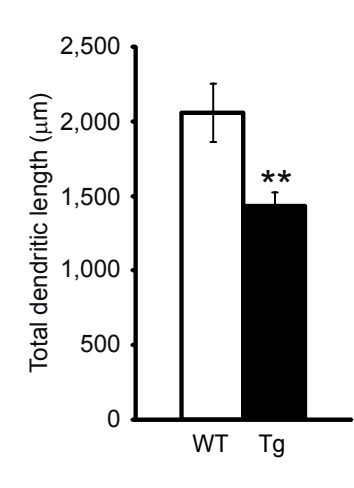


D'Amelio et al., Figure 2

a

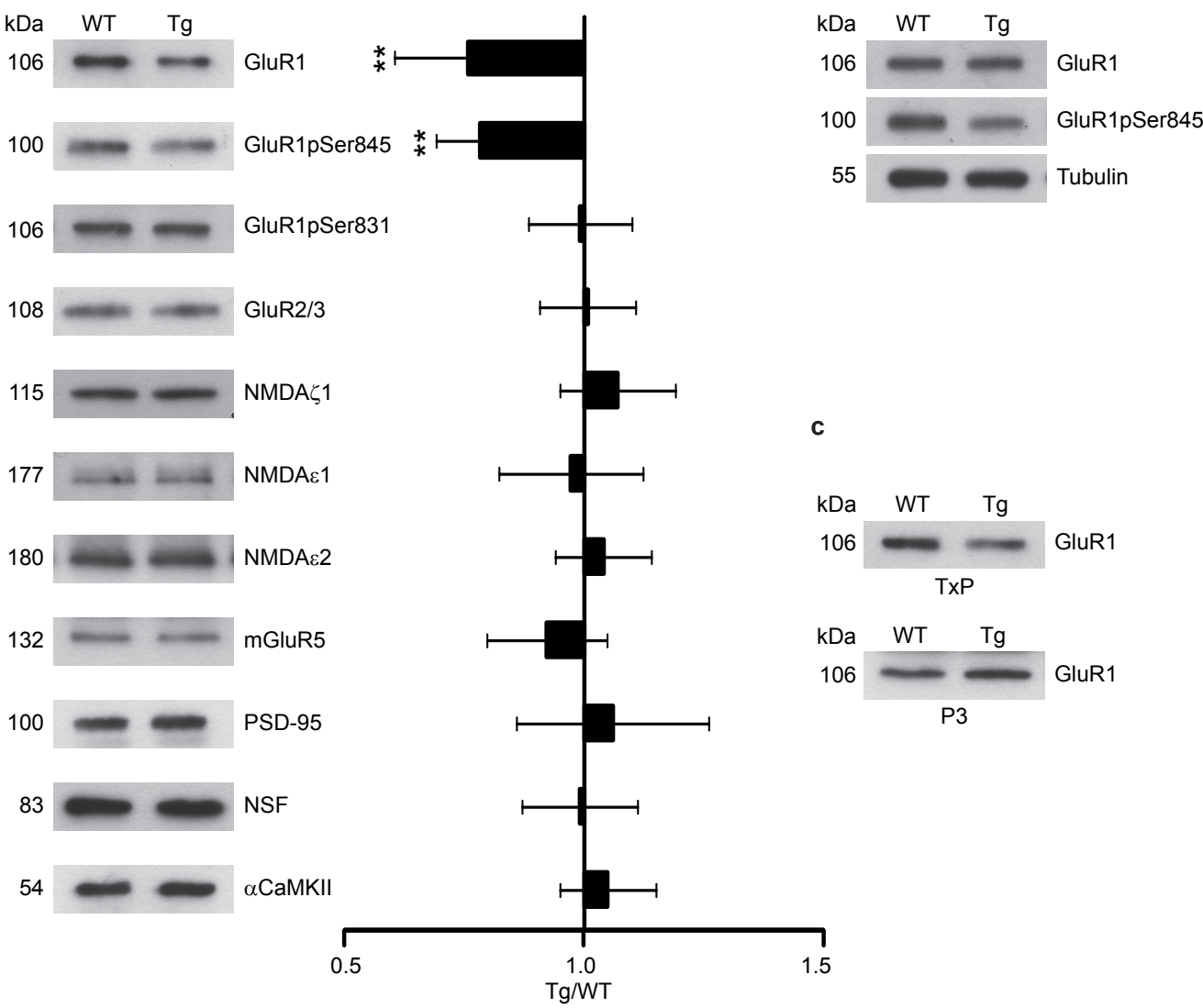

b
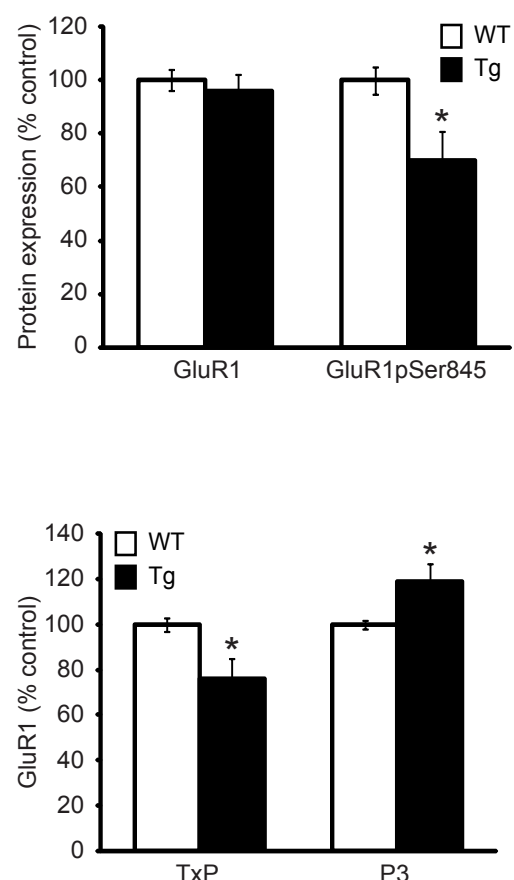
D'Amelio et al., Figure 3

a
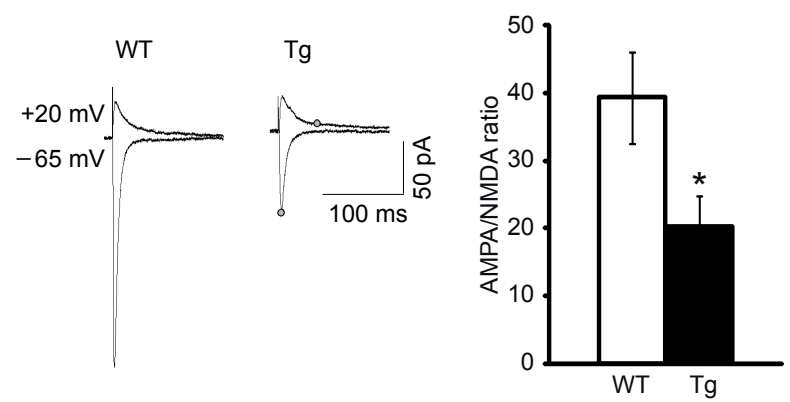

b

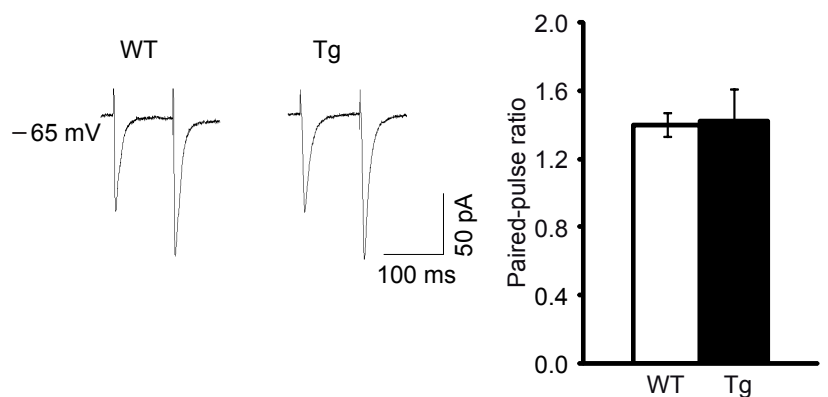

c

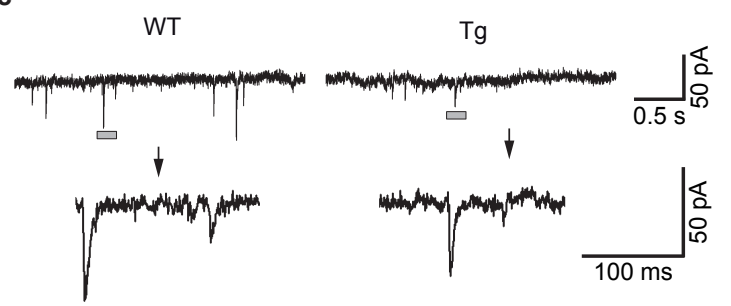

e
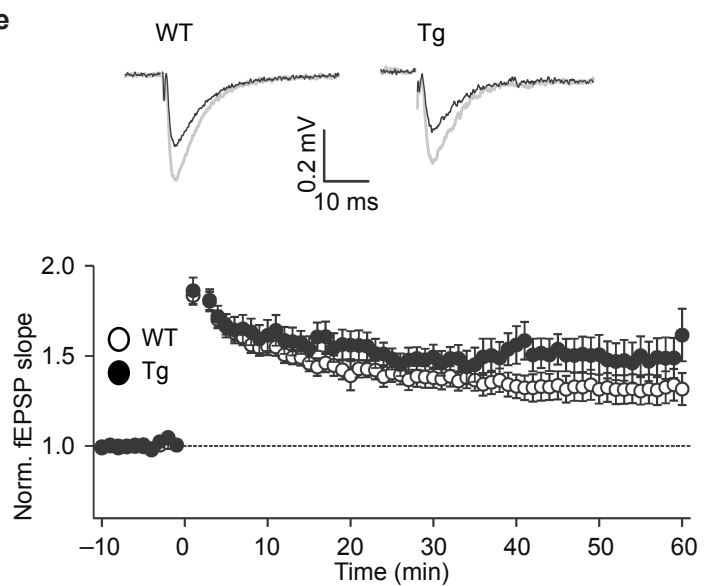
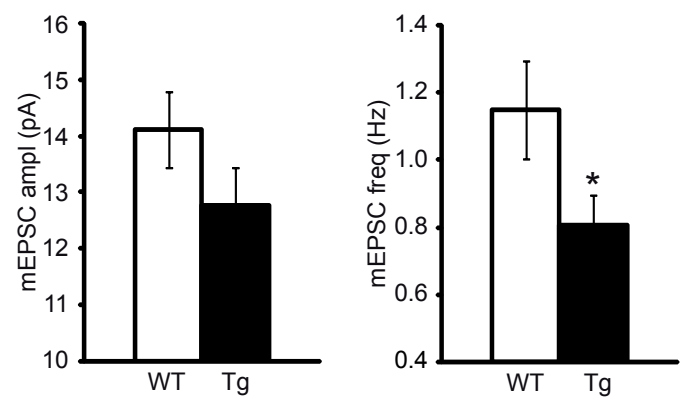

f
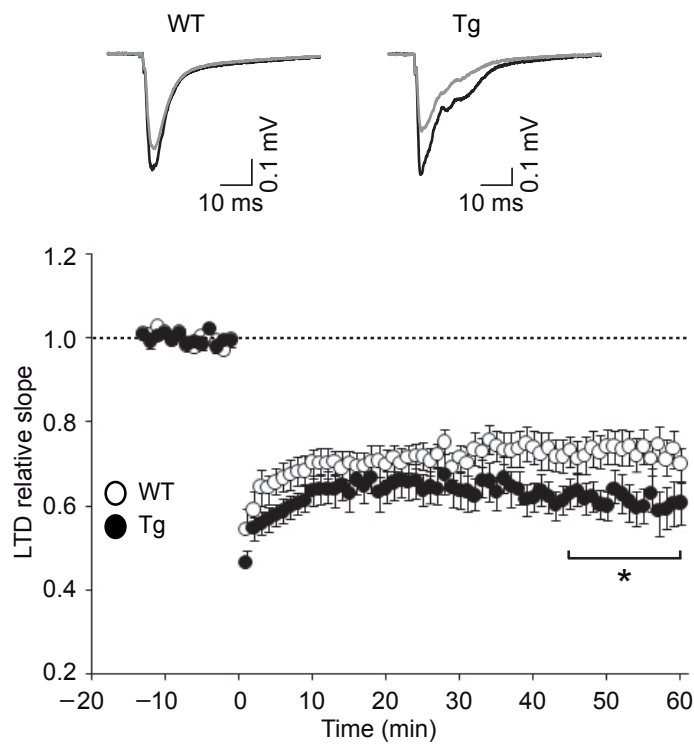


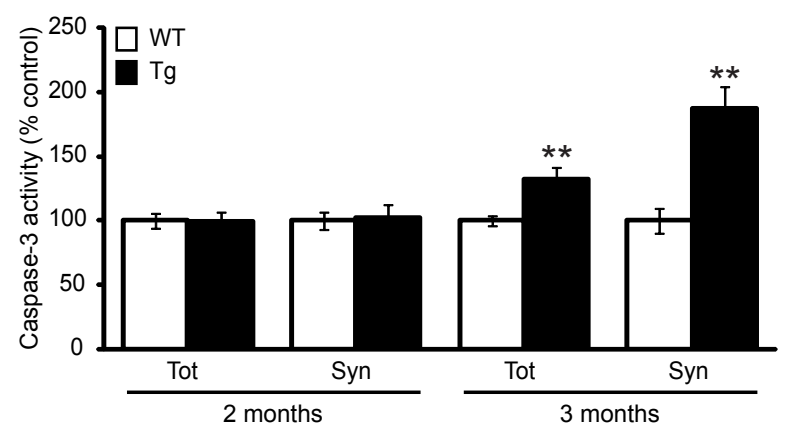

C
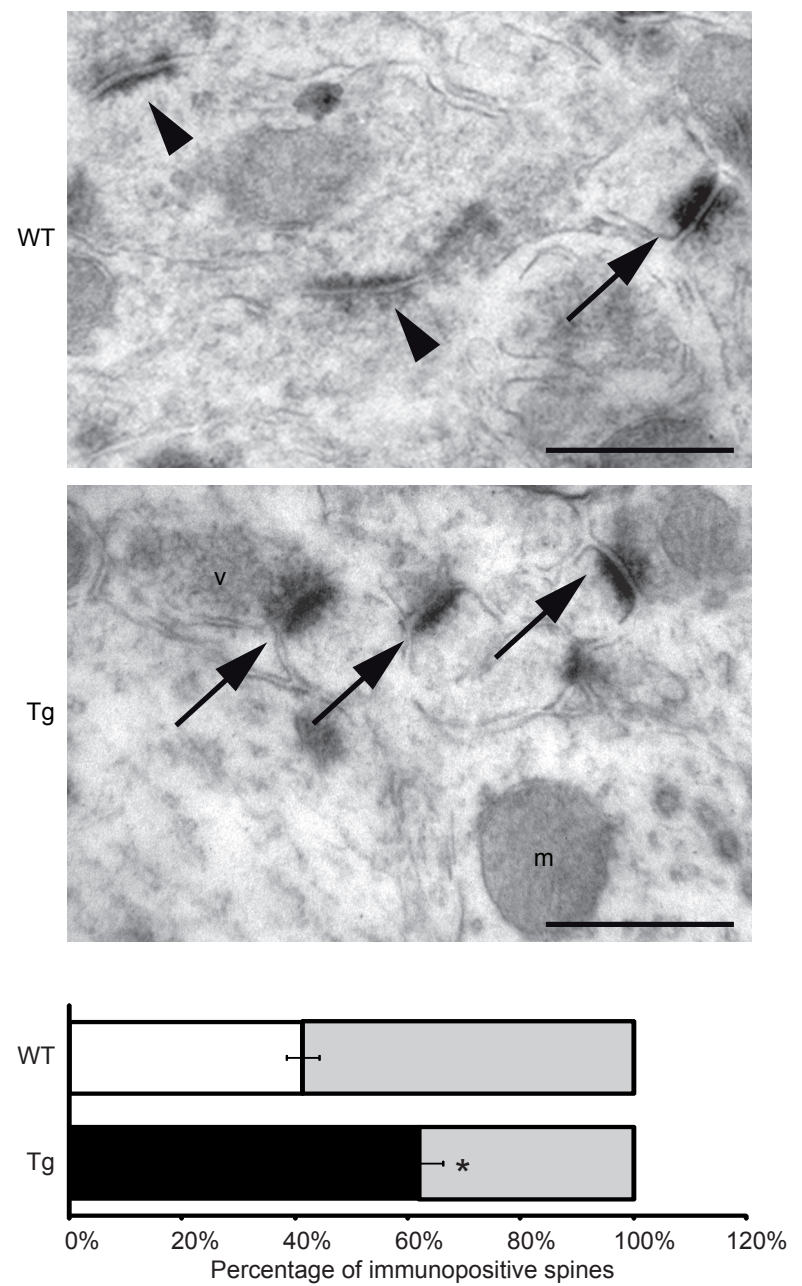

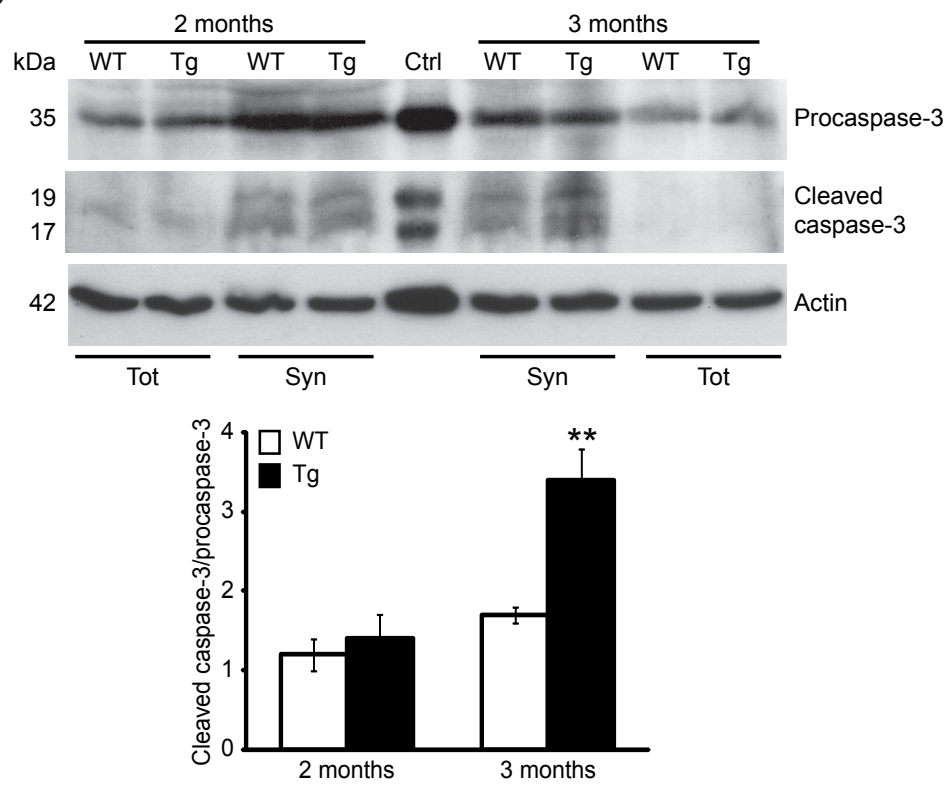

d
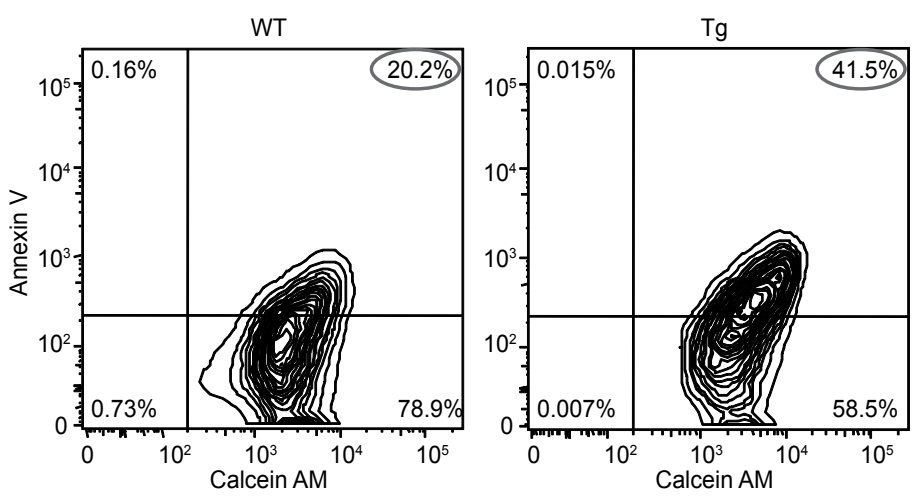

e

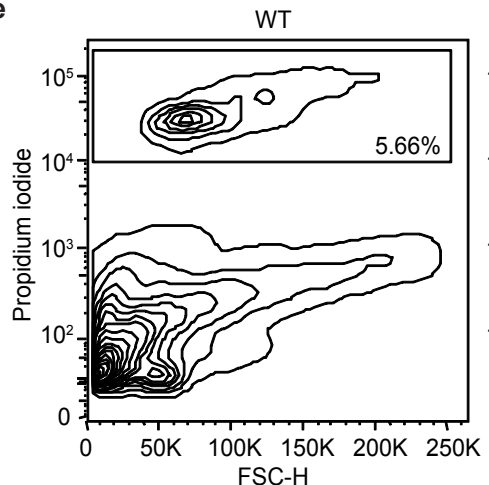



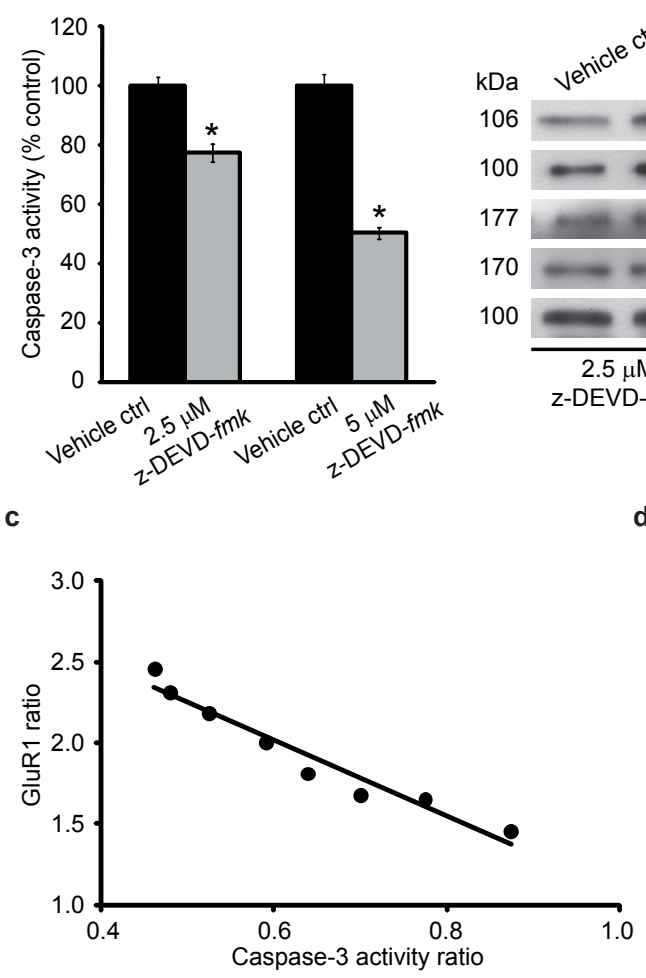

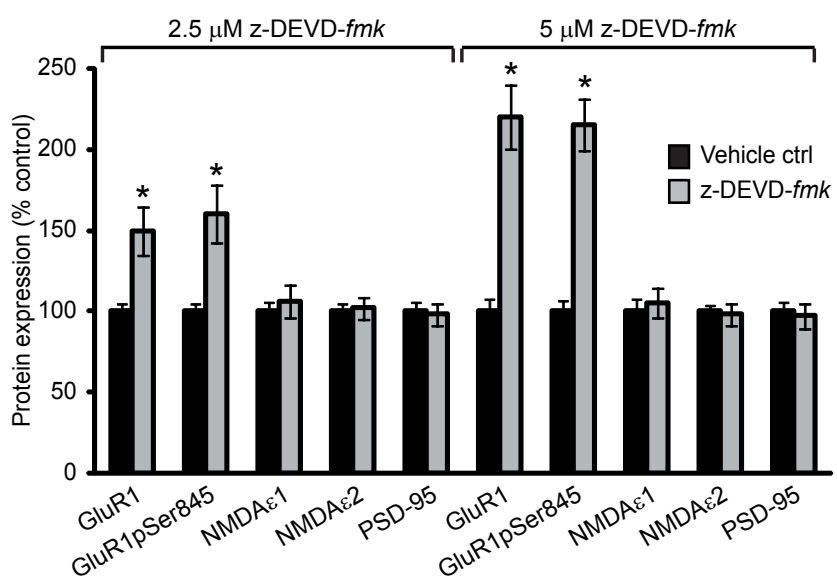

d

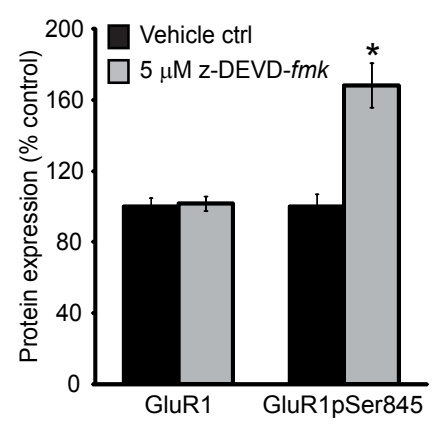

e
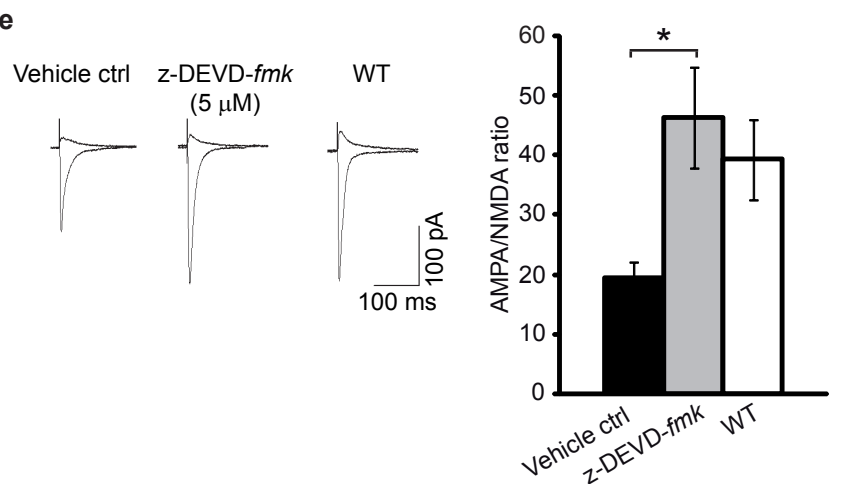

f

Vehicle ctrl z-DEVD-fmk WT $(5 \mu \mathrm{M})$

WT

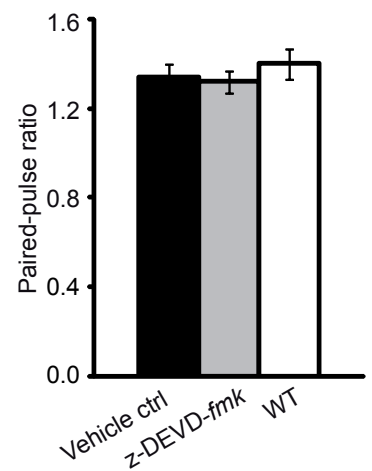


D’Amelio et al., Figure 6

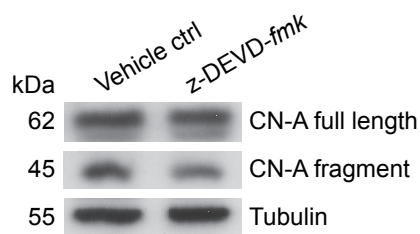

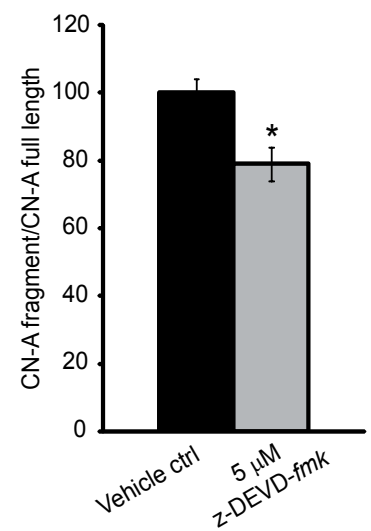

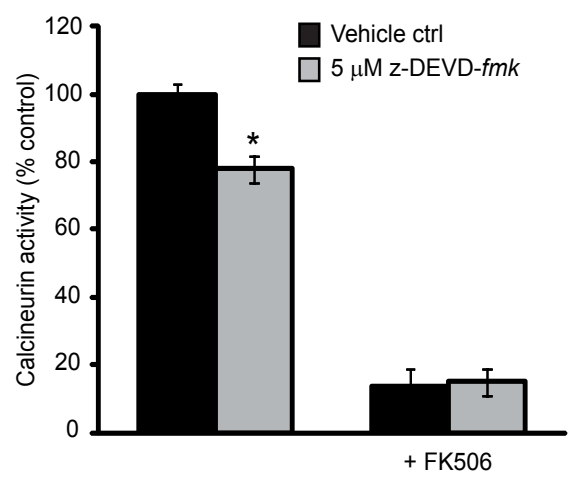


D'Amelio et al., Figure 7

a
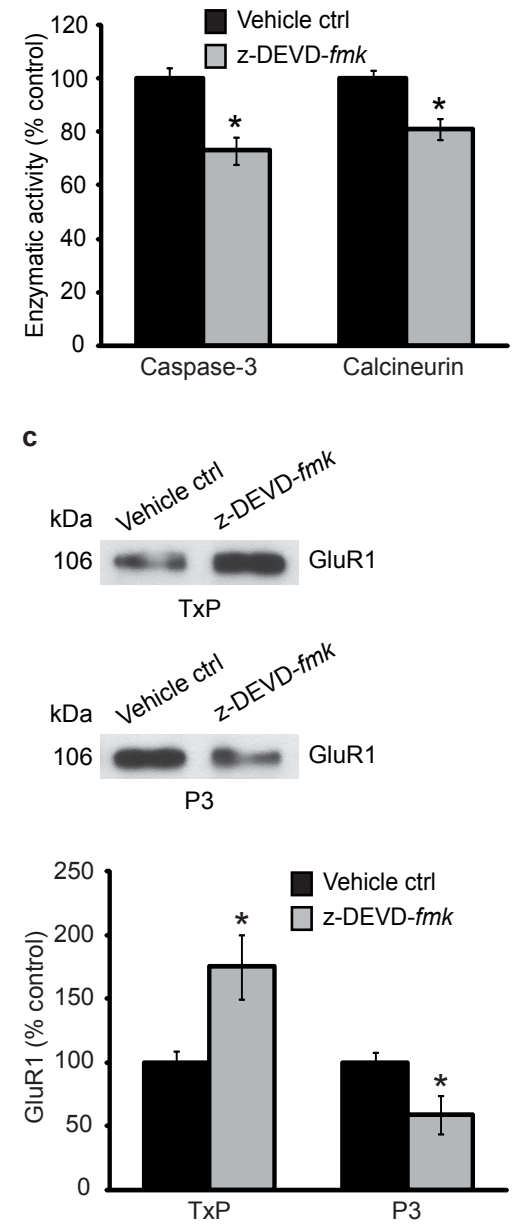

e

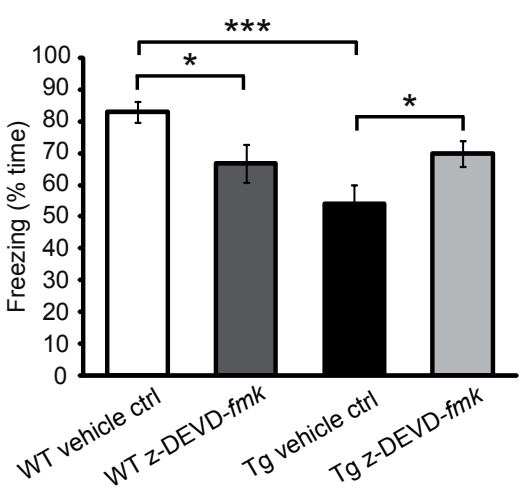

b

d
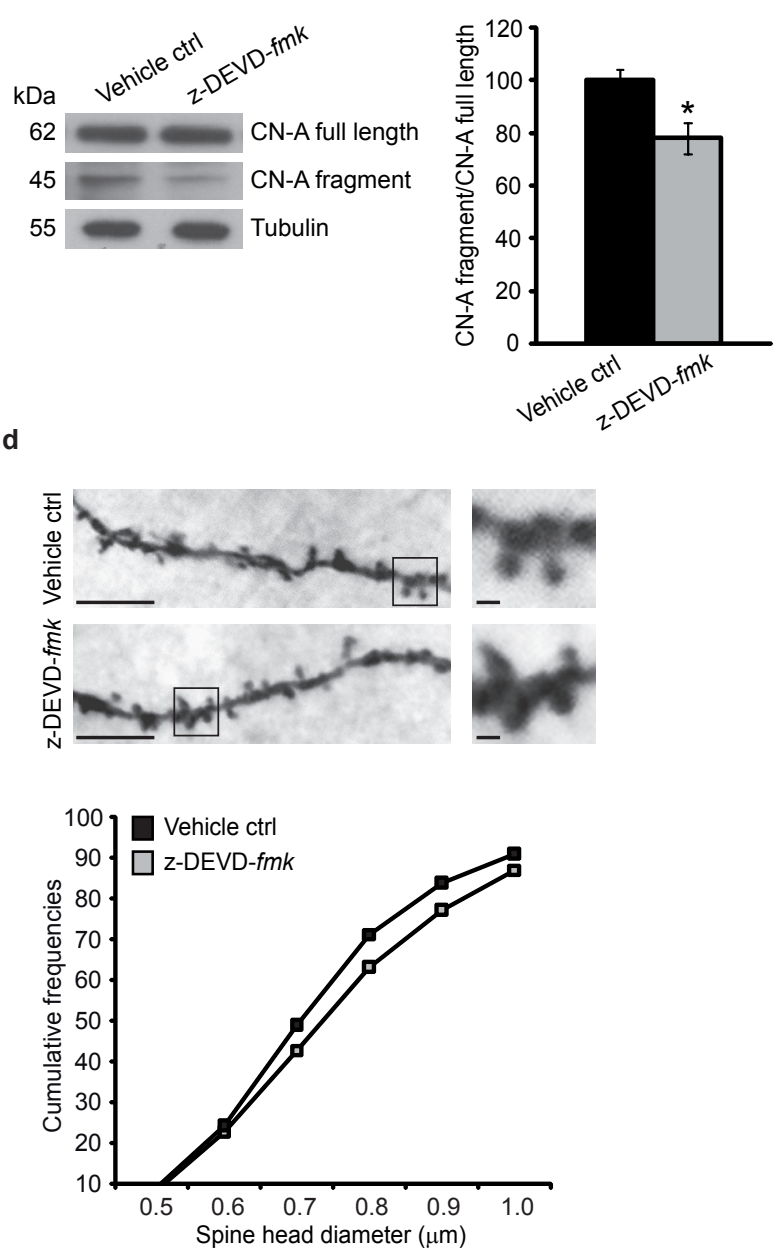